\title{
The Extended Generalized Gamma Geometric Distribution
}

\author{
Juliano Bortolini ${ }^{1}$, Marcelino A. R. Pascoa ${ }^{1}$, Renato Ribeiro de Lima ${ }^{2} \&$ Anderson C. S. Oliveira $^{1}$ \\ ${ }^{1}$ Departamento de Estatística, Universidade Federal de Mato Grosso, Cuiabá, Brazil \\ 2 Departamento de Ciências Exatas, Universidade Federal de Lavras, Lavras, Brazil \\ Correspondence: Marcelino Pascoa, Departamento de Estatística, Universidade Federal de Mato Grosso, Av. Fernando \\ Corrêa da Costa, 2367 - ZIP CODE 78060-900 - Cuiabá (MT), Brazil. E-mail: marcelino.pascoa@ gmail.com
}

Received: March 10, 2017 Accepted: May 22, 2017 Online Published: June 23, 2017

doi:10.5539/ijsp.v6n4p48 URL: https://doi.org/10.5539/ijsp.v6n4p48

\begin{abstract}
We propose and study the so-called extended generalized gamma geometric distribution. The proposed distribution has five parameters and it can be accommodate increasing, decreasing, bathtub and unimodal shaped hazard functions. The new distribution has a large number of well-known lifetime special sub-models such as the generalized gamma geometric, Weibull geometric, gamma geometric, exponential geometric, Rayleigh geometric, half-normal geometric among others. We provide a mathematical treatment of the new distribution including explicit expressions for moments, moment generating function, mean deviations, reliability and order statistics. The method of maximum likelihood and a Bayesian procedure are adopted for estimating the model parameters. Finally, an application of the new distribution is illustrated in a real data sets.
\end{abstract}

Keywords: Generalized gamma distribution, Weibull geometric distribution, lifetime distribution, maximum likelihood estimation, bimodality

\section{Introduction}

Stacy (1962) introduced the generalized gamma (GG) distribution that is an extensive family that contains a varieties of special sub-models, including the gamma, Weibull, log normal and Maxwell distributions, among others. This distribution is useful for modeling lifetime data and for modeling phenomenon with different types of hazard rate function as well as monotonically increasing and decreasing, in the form of bathtub and unimodal (Cox et al., 2007).

Several distributions based on extensions or mixtures of the distributions were developed in last years providing more flexibility for modeling survival data. Adamidis and Loukas (1998) introduced a two-parameter distribution with decreasing hazard rate so-called exponential geometric (EG). Silva et al. (2010) proposed the generalized exponential geometric with decreasing, increasing and unimodal hazard rate depending on its parameters. Barreto-Souza et al. (2011) defined the Weibull geometric (WG) which is an extension of the EG distribution and considered for modeling monotone or unimodal hazard rates. Cordeiro et al. (2011) introduced the exponentiated generalized gamma distribution, Pascoa et al. (2011) proposed the Kumaraswamy generalized gamma and Cordeiro et al. (2013b) defined the beta-Weibull geometric.

Ortega et al. (2011), following the idea of Adamidis and Loukas (1998) for a process of mixing distributions, presented the generalized gamma geometric (GGG) distribution with four parameters that generalizes a number of well-known special lifetime models such as the GG, EG and WG, among others. The GGG distribution can also be obtained by geometric generated family of distributions, that is a special case of the well-known Marshall-Olkin family of distributions proposed by Marshall and Olkin (1997).

The GGG distribution has monotonically increasing and decreasing, in the form of bathtub and unimodal hazard rate. However, this distribution and its sub-models does not provide a reasonable parametric fit for some practical applications where data may be bimodal shape.

In this work we propose so-called the extended generalized gamma geometric (denoted with the prefix "ExGGG" for short) distribution with five parameters and derive some of its properties with the hope that it will attract wider applications in reliability, engineering and in other areas of research. We are motivated to study the ExGGG distribution because of the wide usage of the GGG distribution and their sub-models in survival analysis. It is also suitable for testing goodness-of-fit of some special sub-models, such as the GGG, GG, WG, EG, Weibull and exponential distributions. Furthermore, the current extension provides density and hazard rate functions with great flexibility for to model complex data in a great variety of applications including the bimodal, skewed and heavy-tailed cases.

The paper is outlined as follows. In Section 2, we define the ExGGG distribution and some of its submodels. Further, we derive useful expansions for its density function. In Section 4 we obtain two alternative expansions for the moments. 
In Section 5 we provide an explicit expression for the moment generating function. The mean deviations are determined in Section 6. The reliability is derived in Section 7. In Section 8 we derive the density function of the ith order statistic. Maximum likelihood method and Bayesian approach for the parameter model are discussed in Section 9. The usefulness of the new model is illustrated by means of an application to real data in Section 10. Some conclusions are offered in Section 11.

\section{The ExGGG Distribution}

The distribution GGG with four parameters $\alpha>0, \tau>0, k>0$ and $p \in(0,1)$, defined by Ortega et al. (2011), has the probability density function (pdf) given by

$$
g(x)=\frac{\tau(1-p)}{\alpha \Gamma(k)}\left(\frac{x}{\alpha}\right)^{\tau k-1} \exp \left[-\left(\frac{x}{\alpha}\right)^{\tau}\right]\left(1-p\left\{1-\gamma_{1}\left[k,\left(\frac{x}{\alpha}\right)^{\tau}\right]\right\}\right)^{-2},
$$

where $x>0, \gamma(k, x)=\int_{0}^{x} w^{k-1} e^{-w} d w$ is the incomplete gamma function, $\Gamma(k)=\int_{0}^{\infty} w^{k-1} e^{-w} d w$ is the gamma function and $\gamma_{1}(k, x)=\gamma(k, x) / \Gamma(k)$ is the incomplete gamma function ratio.

The cumulative density function (cdf) of the GGG distribution is

$$
G(x)=\frac{\gamma_{1}\left[k,\left(\frac{x}{\alpha}\right)^{\tau}\right]}{1-p\left\{1-\gamma_{1}\left[k,\left(\frac{x}{\alpha}\right)^{\tau}\right]\right\}}, \quad x>0 .
$$

Let $G(x)$ be a cdf, the extended class of distributions (also referred to as the Lehmann type II class of distributions) presented by Cordeiro et al. (2013a) corresponding to $G(x)$ is defined by $F(x)=1-[1-G(x)]^{\lambda}$, where $\lambda$ is a positive real number. Hence, the cdf of the ExGGG with five parameters $\alpha>0, \tau>0, k>0, \lambda>0$ and $p \in(0,1)$ has the form

$$
F(x)=1-\left\{1-\frac{\gamma_{1}\left[k,\left(\frac{x}{\alpha}\right)^{\tau}\right]}{1-p\left\{1-\gamma_{1}\left[k,\left(\frac{x}{\alpha}\right)^{\tau}\right]\right\}}\right\}^{\lambda}, x>0
$$

and the pdf is given by

$$
\begin{aligned}
f(x) & =\frac{\lambda \tau(1-p)}{\alpha \Gamma(k)}\left(\frac{x}{\alpha}\right)^{\tau k-1} \exp \left[-\left(\frac{x}{\alpha}\right)^{\tau}\right]\left(1-p\left\{1-\gamma_{1}\left[k,\left(\frac{x}{\alpha}\right)^{\tau}\right]\right\}\right)^{-2} \\
& \times\left\{1-\gamma_{1}\left[k,\left(\frac{x}{\alpha}\right)^{\tau}\right]\left(1-p\left\{1-\gamma_{1}\left[k,\left(\frac{x}{\alpha}\right)^{\tau}\right]\right\}\right)^{-1}\right\}^{\lambda-1}
\end{aligned}
$$

A random variable $X$ having pdf (3) is denoted by $\operatorname{ExGGG}(\alpha, \tau, k, p, \lambda)$. Clearly, when $\lambda=1$ we have GGG distribution. Some distributions are obtained from (3) as particular cases, for example, when $k=1$ we have the extended Weibull geometric (ExWG), which is a new distribution, for $k=\lambda=1$ we have the WG distribution, for $\tau=k=\lambda=1$, we obtain the EG distribution. The GG distribution is the limiting distribution (the limit is defined in terms of the convergence in distribution) of the ExGGG distribution when $p \rightarrow 0^{+}$and $\lambda=1$. On the other hand, if $p \rightarrow 1^{-}$, we obtain the distribution of a random variable $Y$ such that $P(Y=0)=1$. Some important ExGGG sub-models are listed in Table 2 .

The survival and hazard rate functions corresponding to (2) are

$$
S(x)=\left\{1-\frac{\gamma_{1}\left[k,\left(\frac{x}{\alpha}\right)^{\tau}\right]}{1-p\left\{1-\gamma_{1}\left[k,\left(\frac{x}{\alpha}\right)^{\tau}\right]\right\}}\right\}^{\lambda}
$$

and

$$
h(x)=\frac{\frac{\lambda \tau(1-p)}{\alpha \Gamma(k)}\left(\frac{x}{\alpha}\right)^{\tau k-1} \exp \left[-\left(\frac{x}{\alpha}\right)^{\tau}\right]\left(1-p\left\{1-\gamma_{1}\left[k,\left(\frac{x}{\alpha}\right)^{\tau}\right]\right\}\right)^{-2}}{1-\frac{\gamma_{1}\left[k,\left(\frac{x}{\alpha}\right)^{\tau}\right]}{1-p\left\{1-\gamma_{1}\left[k,\left(\frac{x}{\alpha}\right)^{\tau}\right]\right\}}},
$$

respectively. 
Table 1. Some ExGGG Distributions

\begin{tabular}{llccll}
\hline Distribution & $\tau$ & $\alpha$ & $k$ & $p$ & $\lambda$ \\
\hline Extended Gamma geometric & 1 & $\alpha$ & $k$ & $p$ & $\lambda$ \\
Extended Chi-square geometric & 1 & 2 & $\frac{n}{2}$ & $p$ & $\lambda$ \\
Extended Exponential geometric & 1 & $\alpha$ & 1 & $p$ & $\lambda$ \\
Extended Weibull geometric & $c$ & $\alpha$ & 1 & $p$ & $\lambda$ \\
Extended Rayleigh geometric & 2 & $\sigma \sqrt{2}$ & 1 & $p$ & $\lambda$ \\
Extended Maxwell geometric & 2 & $\sigma \sqrt{2}$ & $\frac{3}{2}$ & $p$ & $\lambda$ \\
Extended Half normal geometric & 2 & $\sigma \sqrt{2}$ & $\frac{1}{2}$ & $p$ & $\lambda$ \\
Extended Generalized gamma & $\tau$ & $\alpha$ & $k$ & $0^{+}$ & $\lambda$ \\
Extended Gamma & 1 & $\alpha$ & $k$ & $0^{+}$ & $\lambda$ \\
Extended Chi-square & 1 & 2 & $\frac{n}{2}$ & $0^{+}$ & $\lambda$ \\
Extended Exponential & 1 & $\alpha$ & 1 & $0^{+}$ & $\lambda$ \\
Extended Weibull & $c$ & $\alpha$ & 1 & $0^{+}$ & $\lambda$ \\
Extended Rayleigh & 2 & $\sigma \sqrt{2}$ & 1 & $0^{+}$ & $\lambda$ \\
Extended Maxwell & 2 & $\sigma \sqrt{2}$ & $\frac{3}{2}$ & $0^{+}$ & $\lambda$ \\
Extended Half normal & 2 & $\sigma \sqrt{2}$ & $\frac{1}{2}$ & $0^{+}$ & $\lambda$ \\
Generalized gamma geometric & $\tau$ & $\alpha$ & $k$ & $p$ & 1 \\
Gamma geometric & 1 & $\alpha$ & $k$ & $p$ & 1 \\
Chi-square geometric & 1 & 2 & $\frac{n}{2}$ & $p$ & 1 \\
Exponential geometric & 1 & $\alpha$ & 1 & $p$ & 1 \\
Weibull geometric & $c$ & $\alpha$ & 1 & $p$ & 1 \\
Rayleigh geometric & 2 & $\sigma \sqrt{2}$ & 1 & $p$ & 1 \\
Maxwell geometric & 2 & $\sigma \sqrt{2}$ & $\frac{3}{2}$ & $p$ & 1 \\
Half normal geometric & 2 & $\sigma \sqrt{2}$ & $\frac{1}{2}$ & $p$ & 1 \\
Generalized gamma & $\tau$ & $\alpha$ & $k$ & $0^{+}$ & 1 \\
Gamma & 1 & $\alpha$ & $k$ & $0^{+}$ & 1 \\
Chi-square & 1 & 2 & $\frac{n}{2}$ & $0^{+}$ & 1 \\
Exponential & 1 & $\alpha$ & 1 & $0^{+}$ & 1 \\
Weibull & $c$ & $\alpha$ & 1 & $0^{+}$ & 1 \\
Rayleigh & 2 & $\sigma \sqrt{2}$ & 1 & $0^{+}$ & 1 \\
Maxwell & 2 & $\sigma \sqrt{2}$ & $\frac{3}{2}$ & $0^{+}$ & 1 \\
Half normal & 2 & $\sigma \sqrt{2}$ & $\frac{1}{2}$ & $0^{+}$ & 1 \\
\hline
\end{tabular}

Plots of the ExGGG density and hazard rate function for selected parameter values are given in Figures 1 and 2, respectively. These plots illustrate the versatility of the ExGGG distribution.

Note in Figure 1(a) that the density function of the ExGGG distribution has very flexible shapes, especially bimodal. This is a great advantage of the distribution proposed in relation to its sub-models because none has bimodal density. The Figure 1 indicate that $\alpha$ is a scale parameter, whereas $\tau, k, p$ and $\lambda$ are shape parameters. The hazard rate function also presents some peculiar shapes. For instance, the blue hazard rate function in Figure 2(a) is initially increasing and then decreasing and finally increasing again.

The ExGGG distribution have an attractive physical interpretation whenever $\lambda$ is positive integer. Consider a device made of $\lambda$ components independent and identically distributed according to $G(x)(1)$ in a series system. The device fails if any component fails. Let $X_{1}, \cdots, X_{\lambda}$ denote the lifetimes of the components, with common cdf $G(x)$. Let $X$ denote the lifetime of the device. Thus, the cdf $F(x)$ of $X$ is

$$
\begin{aligned}
F(x) & =P(X \leq x)=1-P(X>x)=1-P\left(X_{1}>x, \cdots, X_{\lambda}>x\right) \\
& =1-P\left(X_{1}>x\right)^{\lambda}=1-\left[1-P\left(X_{1} \leq x\right)\right]^{\lambda} \\
& =1-[1-G(x)]^{\lambda} .
\end{aligned}
$$

So, the lifetime of the device obeys the ExGGG distribution. 
(a)

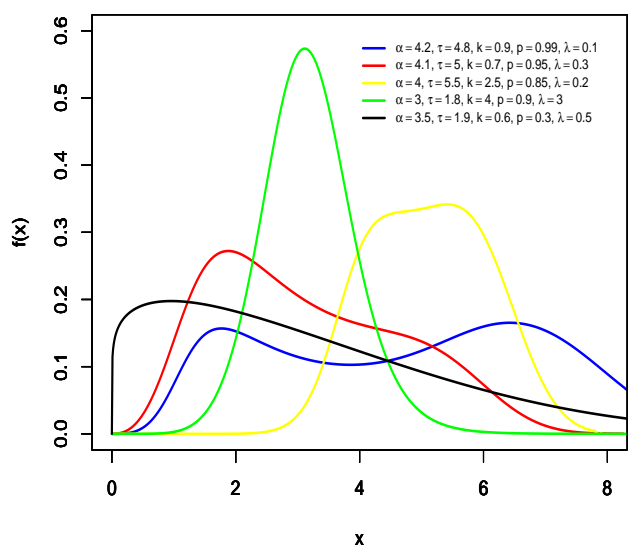

(c)

$\operatorname{ExGGG}(\alpha=4, \tau, \mathrm{k}=0.7, \mathrm{p}=0.95, \lambda=0.4)$

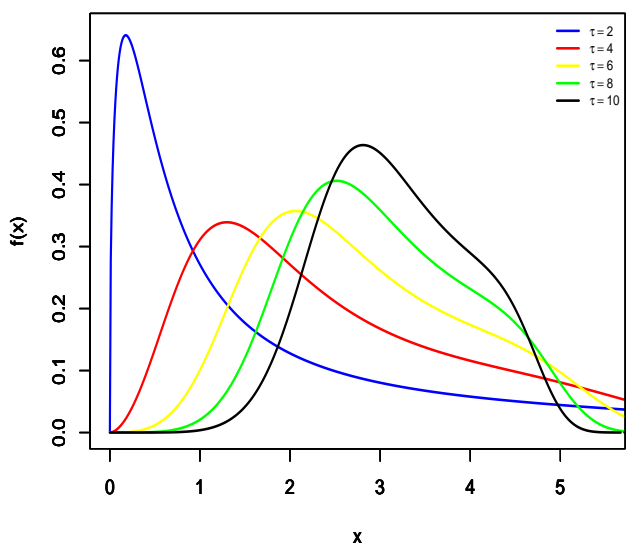

(e)

$\operatorname{ExGGG}(\alpha=3, \tau=4, \mathrm{k}=1.25, \mathrm{p}, \lambda=0.2)$

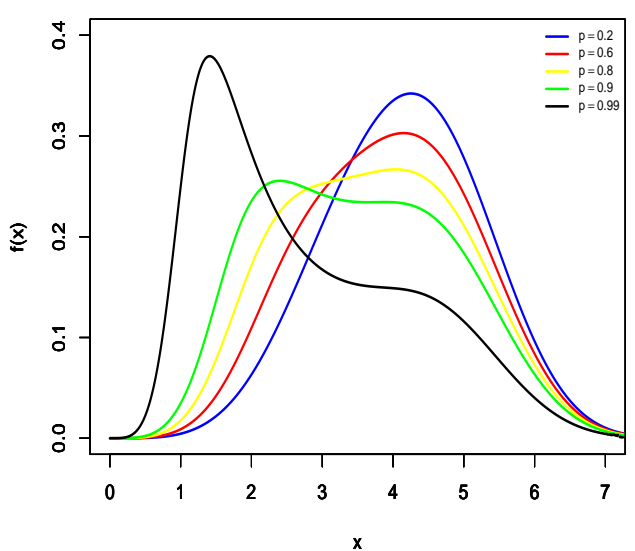

(b)

$\operatorname{ExGGG}(\alpha, \tau=1.2, \mathrm{k}=3, \mathrm{p}=0.2, \lambda=1.2)$

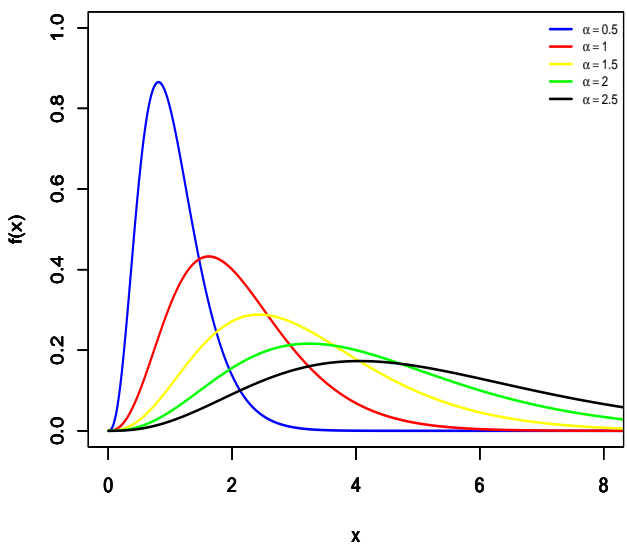

(d)

$\operatorname{ExGGG}(\alpha=2, \tau=2.5, \mathrm{k}, \mathrm{p}=0.9, \lambda=0.15)$



(f)

$\operatorname{ExGGG}(\alpha=3.25, \tau=5, k=0.9, p=0.95, \lambda)$

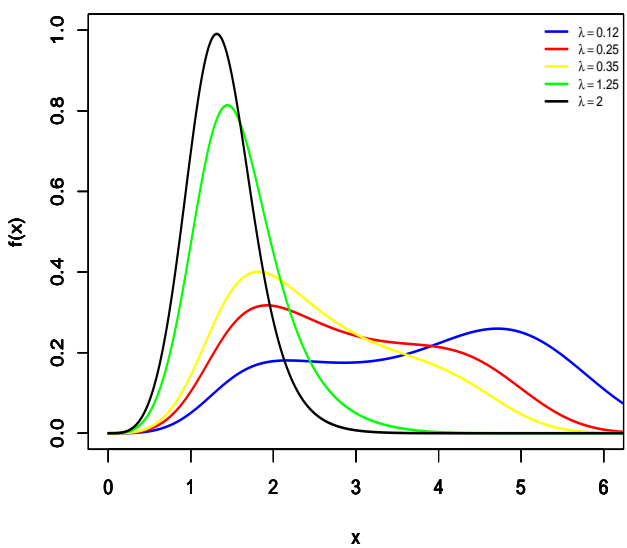

Figure 1. Plots of the ExGGG density for some parameter values. 
(a)

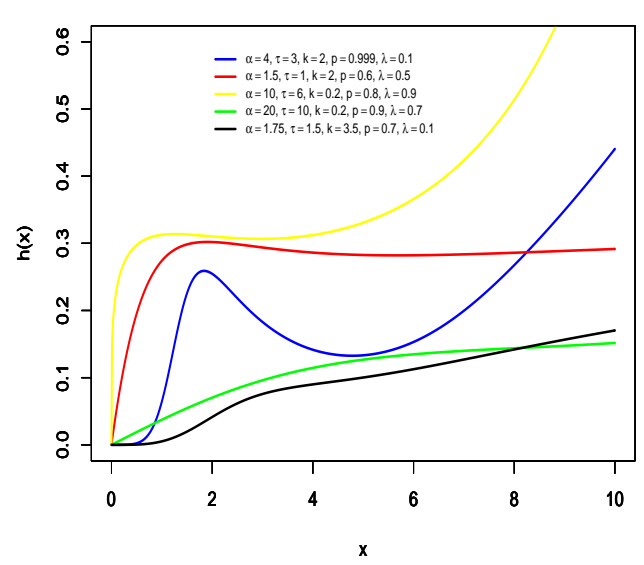

(c)

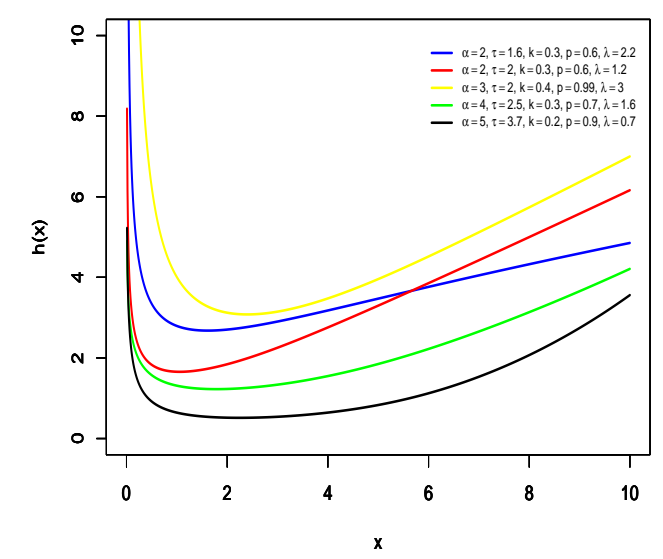

(b)

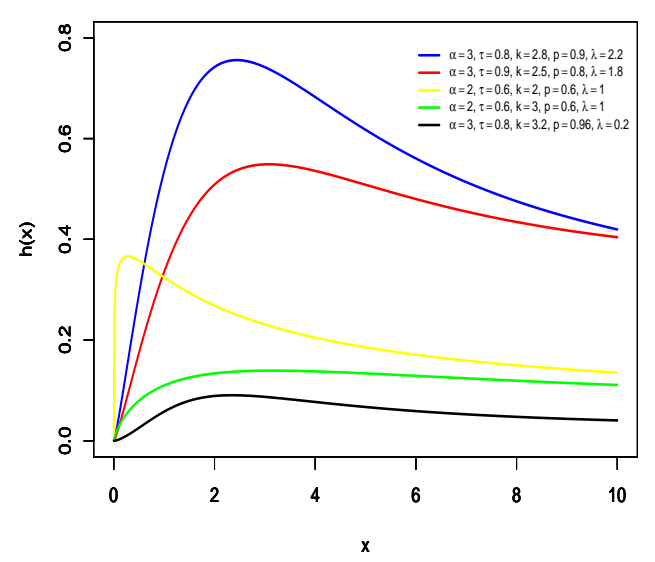

(d)

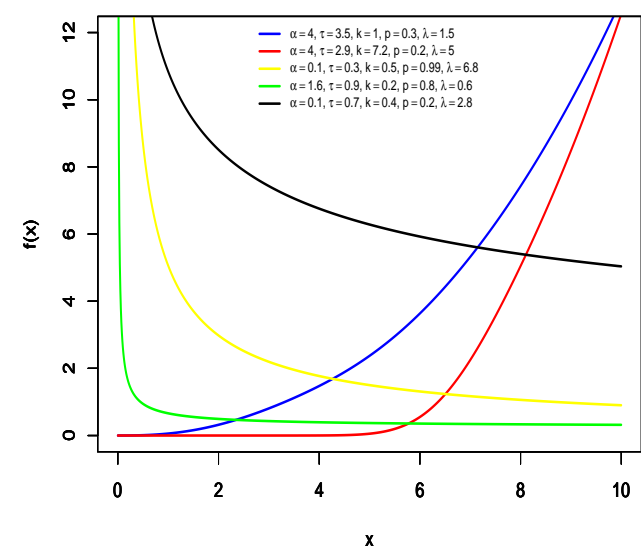

Figure 2. The ExGGG hazard rate function. (a) Plots of the hazard rate function for some parameter values. (b) Unimodal hazard rate function. (c) Bathtub hazard rate function. (d) Increasing and decreasing hazard rate function.

\section{Expansion of the Density Function}

Now, we demonstrate that the density function (3) can be expressed as a linear combination of GG density functions. This result is important to provide mathematical properties of the ExGGG distribution directly from properties of the GG distribution.

Let $g_{\alpha, \tau, k}(x)$ be the density function of the $\mathrm{GG}(\alpha, \tau, k)$ distribution given by

$$
g_{\alpha, \tau, k}(x)=\frac{\tau}{\alpha \Gamma(k)}\left(\frac{x}{\alpha}\right)^{\tau k-1} \exp \left[-\left(\frac{x}{\alpha}\right)^{\tau}\right], \quad x>0 .
$$

For $|z|<1$ and $\rho \in \mathbb{R}$, we consider the power series

$$
(1-z)^{\rho}=\sum_{j=0}^{\infty}(-1)^{j}\left(\begin{array}{l}
\rho \\
j
\end{array}\right) z^{j},
$$

where $\left(\begin{array}{l}\rho \\ j\end{array}\right)=\Gamma(\rho+1) /[\Gamma(\rho-j+1) j !]$. 
Considering (4) in (3), the pdf of the $\operatorname{ExGGG}(\alpha, \tau, k, p, \lambda)$ can be written as

$$
\begin{aligned}
f(x) & =\frac{\lambda \tau(1-p)}{\alpha \Gamma(k)}\left(\frac{x}{\alpha}\right)^{\tau k-1} \exp \left[-\left(\frac{x}{\alpha}\right)^{\tau}\right]\left(1-p\left\{1-\gamma_{1}\left[k,\left(\frac{x}{\alpha}\right)^{\tau}\right]\right\}\right)^{-2} \\
& \times \sum_{j=0}^{\infty}(-1)^{j}\left(\begin{array}{c}
\lambda-1 \\
j
\end{array}\right) \gamma_{1}\left[k,\left(\frac{x}{\alpha}\right)^{\tau}\right]^{j}\left(1-p\left\{1-\gamma_{1}\left[k,\left(\frac{x}{\alpha}\right)^{\tau}\right]\right\}\right)^{-j} .
\end{aligned}
$$

Grouping common terms, using (4) and binomial expansion, we have that

$$
f(x)=\frac{\lambda \tau(1-p)}{\alpha \Gamma(k)}\left(\frac{x}{\alpha}\right)^{\tau k-1} \exp \left[-\left(\frac{x}{\alpha}\right)^{\tau}\right] \sum_{j, l=0}^{\infty} \sum_{m=0}^{l}(-1)^{j+l+m}\left(\begin{array}{c}
\lambda-1 \\
j
\end{array}\right)\left(\begin{array}{c}
-(j+2) \\
l
\end{array}\right)\left(\begin{array}{l}
l \\
m
\end{array}\right) p^{l} \gamma_{1}\left[k,\left(\frac{x}{\alpha}\right)^{\tau}\right]^{j+m}
$$

We can substitute $\sum_{j, l=0}^{\infty} \sum_{m=0}^{l}$ for $\sum_{j, m=0}^{\infty} \sum_{l=m}^{\infty}$ to obtain

$$
f(x)=\frac{\lambda \tau(1-p)}{\alpha \Gamma(k)}\left(\frac{x}{\alpha}\right)^{\tau k-1} \exp \left[-\left(\frac{x}{\alpha}\right)^{\tau}\right] \sum_{j, m=0}^{\infty} s_{j, m}(\lambda, p) \gamma_{1}\left[k,\left(\frac{x}{\alpha}\right)^{\tau}\right]^{j+m},
$$

where

$$
s_{j, m}(\lambda, p)=\sum_{l=m}^{\infty}(-1)^{j+l+m}\left(\begin{array}{c}
\lambda-1 \\
j
\end{array}\right)\left(\begin{array}{c}
-(j+2) \\
l
\end{array}\right)\left(\begin{array}{l}
l \\
m
\end{array}\right) p^{l}
$$

Therefore, using the result (28) (givin in Appendix A) in the expression (5), the pdf $f(x)$ can be written as a linear combination of the distribution GG, in the form:

$$
f(x)=\sum_{j, m, q=0}^{\infty} w_{j, m, q}(k, p, \lambda) g_{\alpha, \tau, k} \cdot(x), \quad x>0
$$

where $k^{\bullet}=k(j+m+1)+q, g_{\alpha, \tau, k^{\bullet}}(x)$ has distribution $\mathrm{GG}\left(\alpha, \tau, k^{\bullet}\right)$ and the weightings $w_{j, m, q}(k, p, \lambda)$ are given by

$$
w_{j, m, q}(k, p, \lambda)=\lambda(1-p) s_{j, m}(\lambda, p) c_{j+m, q} \frac{\Gamma\left(k^{\bullet}\right)}{\Gamma(k)^{j+m+1}},
$$

and the coefficients $c_{j+m, q}$ are determined from the recurrence relation (27) (Appendix A).

Expression (7) shows that the density function ExGGG distribution can be written in terms of a linear combination of densities GG.

\section{Moments}

Some important features of a distribution such as dispersion, skewness and kurtosis can be studied through its moments. This section we obtain two alternative expansions for the moments of the ExGGG distribution. Initially, we know that the $r t h$ ordinary moment of the $\mathrm{GG}(\alpha, \tau, k)$ distribution, denoted by $\mu_{r, G G}^{\prime}$, is

$$
\mu_{r, G G}^{\prime}=\frac{\alpha^{r} \Gamma(k+r / \tau)}{\Gamma(k)} .
$$

Now, follows from expressions (7) and (9), the $r$ th moment ordinary of the $\operatorname{ExGGG}(\alpha, \tau, k, p, \lambda)$ is given by

$$
\mu_{r}^{\prime}=\alpha^{r} \sum_{j, m, q=0}^{\infty} w_{j, m, q}(k, p, \lambda) \frac{\Gamma\left(k^{\bullet}+r / \tau\right)}{\Gamma\left(k^{\bullet}\right)} .
$$

The expression (10) depends on the quantities $c_{j+m, q}$ which are obtained recursively by (27).

Another infinite sum representation for $\mu_{r}^{\prime}$ is obtained computing the moment directly, that is

$$
\begin{aligned}
\mu_{r}^{\prime} & =\frac{\lambda \tau(1-p)}{\alpha \Gamma(k)} \int_{0}^{\infty} x^{r}\left(\frac{x}{\alpha}\right)^{\tau k-1} \exp \left[-\left(\frac{x}{\alpha}\right)^{\tau}\right]\left(1-p\left\{1-\gamma_{1}\left[k,\left(\frac{x}{\alpha}\right)^{\tau}\right]\right\}\right)^{-2} \\
& \times\left\{1-\gamma_{1}\left[k,\left(\frac{x}{\alpha}\right)^{\tau}\right]\left(1-p\left\{1-\gamma_{1}\left[k,\left(\frac{x}{\alpha}\right)^{\tau}\right]\right\}\right)^{-1}\right\}^{\lambda-1} d x
\end{aligned}
$$


Setting $y=\left(\frac{x}{\alpha}\right)^{\tau}$ in the last expression,

$$
\mu_{r}^{\prime}=\frac{\lambda(1-p) \alpha^{r}}{\Gamma(k)} \int_{0}^{\infty} y^{k+\frac{r}{\tau}-1} \exp (-y)\left\{1-p\left[1-\gamma_{1}(k, y)\right]\right\}^{-2}\left\{1-\gamma_{1}(k, y)\left\{1-p\left[1-\gamma_{1}(k, y)\right]\right\}^{-1}\right\}^{\lambda-1} d y .
$$

Considering (4) in (11) twice conveniently, we have that

$$
\mu_{r}^{\prime}=\frac{\lambda(1-p) \alpha^{r}}{\Gamma(k)} \int_{0}^{\infty} y^{k+\frac{r}{\tau}-1} \exp (-y) \sum_{j, l=0}^{\infty}(-1)^{j+l}\left(\begin{array}{c}
\lambda-1 \\
j
\end{array}\right)\left(\begin{array}{c}
-(j+2) \\
l
\end{array}\right) \gamma_{1}(k, y)^{j} p^{l}\left[1-\gamma_{1}(k, y)\right]^{l} d y .
$$

Using the binomial expansion in the term $\left[1-\gamma_{1}(k, y)\right]^{l}$, the last the expression is rewritten as

$$
\mu_{r}^{\prime}=\frac{\lambda(1-p) \alpha^{r}}{\Gamma(k)} \sum_{j, l=0}^{\infty} \sum_{m=0}^{l}(-1)^{j+l+m}\left(\begin{array}{c}
\lambda-1 \\
j
\end{array}\right)\left(\begin{array}{c}
-(j+2) \\
l
\end{array}\right)\left(\begin{array}{l}
l \\
m
\end{array}\right) p^{l} \int_{0}^{\infty} y^{k+\frac{r}{\tau}-1} \exp (-y) \gamma_{1}(k, y)^{j+m} d y .
$$

Replacing $\sum_{j, l=0}^{\infty} \sum_{m=0}^{l}$ for $\sum_{j, m=0}^{\infty} \sum_{l=m}^{\infty}$ in the expression (12), we have

$$
\mu_{r}^{\prime}=\frac{\lambda(1-p) \alpha^{r}}{\Gamma(k)} \sum_{j, m=0}^{\infty} \sum_{l=m}^{\infty}(-1)^{j+l+m}\left(\begin{array}{c}
\lambda-1 \\
j
\end{array}\right)\left(\begin{array}{c}
-(j+2) \\
l
\end{array}\right)\left(\begin{array}{l}
l \\
m
\end{array}\right) p^{l} \int_{0}^{\infty} y^{k+\frac{r}{\tau}-1} \exp (-y) \gamma_{1}(k, y)^{j+m} d y
$$

Therefore $\mu_{r}^{\prime}$ can be rewritten as

$$
\mu_{r}^{\prime}=\frac{\lambda(1-p) \alpha^{r}}{\Gamma(k)} s_{j, m}(\lambda, p) I\left(k+\frac{r}{\tau}, j+m\right),
$$

where $s_{j, m}(\lambda, p)$ is defined by expression (6), and

$$
I\left(k+\frac{r}{\tau}, j+m\right)=\int_{0}^{\infty} y^{k+\frac{r}{\tau}-1} \exp (-y) \gamma_{1}(k, y)^{j+m} d y .
$$

This integral can be determined from expressions (24) and (25) of Nadarajah (2008) in terms of the Lauricella function of type A (Exton, 1978; Aarts, 2000) defined by

$$
F_{A}^{(n)}\left(a ; b_{1}, \ldots, b_{n} ; c_{1}, \ldots, c_{n} ; x_{1}, \ldots, x_{n}\right)=\sum_{m_{1}=0}^{\infty} \cdots \sum_{m_{n}=0}^{\infty} \frac{(a)_{m_{1}+\cdots+m_{n}}\left(b_{1}\right)_{m_{1}} \cdots\left(b_{n}\right)_{m_{n}}}{\left(c_{1}\right)_{m_{1}} \cdots\left(c_{n}\right)_{m_{n}}} \frac{x_{1}^{m_{1}} \cdots x_{n}^{m_{n}}}{m_{1} ! \cdots m_{n} !},
$$

where $(a)_{i}$ is the ascending factorial defined by $(a)_{i}=a(a+1) \cdots(a+i-1)$ assuming $(a)_{0}=1$. Numerical routines for the direct computation of the Lauricella function of type A are available (Exton, 1978; Trott 2006). We obtain

$$
I\left(k+\frac{r}{\tau}, j+m\right)=k^{-(j+m)} \Gamma[r / \tau+k(j+m+1)] \times F_{A}^{(j+m)}(r / \tau+k(j+m+1) ; k, \cdots, k ; k+1, \cdots, k+1 ;-1, \cdots,-1) .
$$

The graphic representations of the skewness and kurtosis measures in terms of $\lambda$ for selected values of $\alpha, \tau, k$ and $p$, are shown in Figure 3.

\section{Moment Generating Function}

Here, we provide two expressions for the mgf of ExGGG distribution based on mgf of GG distribution.

Let $M_{\alpha, \tau, k}(s)=E[\exp (s X)]$ be $\operatorname{mgf}$ of $X \sim G G(\alpha, \tau, k)$. We can write

$$
M_{\alpha, \tau, k}(s)=\frac{\tau}{\alpha^{\tau k} \Gamma(k)} \int_{0}^{\infty} \exp (s x) x^{\tau k-1} \exp \left\{-(x / \alpha)^{\tau}\right\} d x .
$$

Setting $u=x / \alpha$, we have

$$
M_{\alpha, \tau, k}(s)=\frac{\tau}{\Gamma(k)} \int_{0}^{\infty} \exp (\alpha s u) u^{\tau k-1} \exp \left(-u^{\tau}\right) d u
$$


(a)

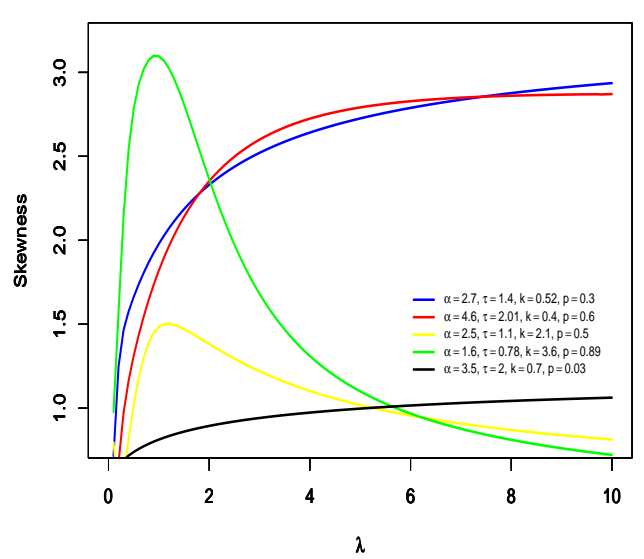

(b)

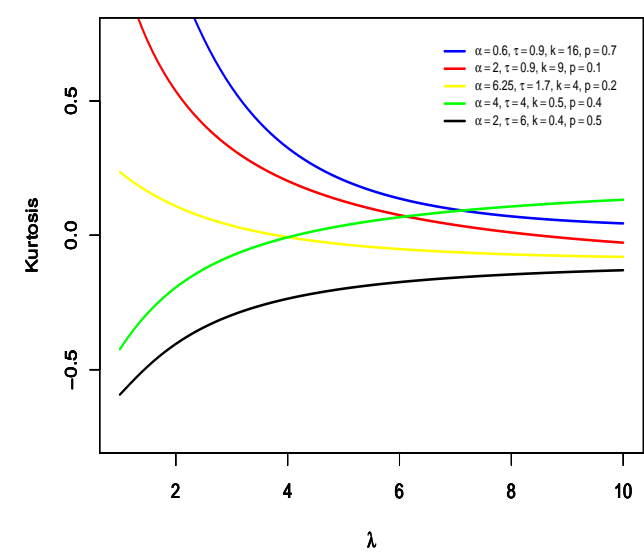

Figure 3. Skewness and kurtosis of the ExGGG distribution as a function of the parameter $\lambda$.

Expanding the first exponential in Taylor series and using $\int_{0}^{\infty} u^{k \tau+d-1} \exp \left(-u^{\tau}\right) d u=\tau^{-1} \Gamma(k+d / \tau)$, we obtain

$$
M_{\alpha, \tau, k}(s)=\frac{1}{\Gamma(k)} \sum_{d=0}^{\infty} \Gamma\left(\frac{d}{\tau}+k\right) \frac{(\alpha s)^{d}}{d !} .
$$

Using the result in (7), the $\operatorname{mgf}$ of $\operatorname{ExGGG}(\alpha, \tau, k, p, \lambda)$ is given by

$$
M(s)=\sum_{j, m, q=0}^{\infty} w_{j, m, q}(k, p, \lambda) M_{\alpha, \tau, k} \cdot(s),
$$

where $k^{\bullet}=k(j+m+1)+q$ and $w_{j, m, q}(k, p, \lambda)$ is given by (8). Therefore,

$$
M(s)=\sum_{j, m, q, d=0}^{\infty} \frac{w_{j, m, q}(k, p, \lambda)}{\Gamma\left(k^{\bullet}\right)} \Gamma\left(\frac{d}{\tau}+k^{\bullet}\right) \frac{(\alpha s)^{d}}{d !} .
$$

However, for $\tau>1$, it can be simplified by considering the Wright generalized hypergeometric function (Wright, 1935) defined by

$$
{ }_{p} \Psi_{q}\left[\begin{array}{c}
\left(\alpha_{1}, A_{1}\right), \cdots,\left(\alpha_{p}, A_{p}\right) \\
\left(\beta_{1}, B_{1}\right), \cdots,\left(\beta_{q}, B_{q}\right)
\end{array} ; x\right]=\sum_{d=0}^{\infty} \frac{\prod_{j=1}^{p} \Gamma\left(\alpha_{j}+A_{j} d\right)}{\prod_{j=1}^{q} \Gamma\left(\beta_{j}+B_{j} d\right)} \frac{x^{d}}{d !}
$$

This function exists if $1+\sum_{j=1}^{q} B_{j}-\sum_{j=1}^{p} A_{j}>0$. Combining the results in (14) to rewrite (13), we have

$$
M_{\alpha, \tau, k}(s)=\frac{1}{\Gamma(k)}{ }_{1} \Psi_{0}\left[\begin{array}{c}
(k, 1 / \tau) \\
-
\end{array} ; \alpha s\right]
$$

Finally, the mgf of ExGGG can be written from expressions (7) and (15) as

$$
M(s)=\sum_{j, m, q=0}^{\infty} \frac{w_{j, m, q}(k, p, \lambda)}{\Gamma\left(k^{\bullet}\right)}{ }_{1} \Psi_{0}\left[\begin{array}{c}
\left(k^{\bullet}, 1 / \tau\right) \\
-
\end{array} ; \alpha s\right] .
$$




\section{Mean Deviations}

The amount of scatter in a population is evidently measured to some extent by the totality of deviations from the mean and median. If $X$ has the ExGGG distribution with density function $f(x)$, we can derive the mean deviations about the mean $\mu_{1}^{\prime}=E(X)$ and about the median $m_{1}$ from the relations

$$
\delta_{1}=\int_{0}^{\infty}\left|x-\mu_{1}^{\prime}\right| f(x) d x \quad \text { and } \quad \delta_{2}=\int_{0}^{\infty}\left|x-m_{1}\right| f(x) d x .
$$

The measures $\delta_{1}$ and $\delta_{2}$ can be expressed as

$$
\delta_{1}=2 \mu_{1}^{\prime} F\left(\mu_{1}^{\prime}\right)-2 I\left(\mu_{1}^{\prime}\right) \quad \text { and } \quad \delta_{2}=\mu_{1}^{\prime}-2 I\left(m_{1}\right),
$$

where $I(s)=\int_{0}^{s} x f(x) d x$ and $F\left(\mu_{1}^{\prime}\right)$ is calculated from (2). The integral $I\left(m_{1}\right)$ can be obtained from expression (7) as

$$
\begin{aligned}
I\left(m_{1}\right) & =\sum_{j, m, q=0}^{\infty} w_{j, m, q}(k, p, \lambda) \int_{0}^{m_{1}} x g_{\alpha, \tau, k}(x) d x \\
& =\sum_{j, m, q=0}^{\infty} w_{j, m, q}(k, p, \lambda) J\left(\alpha, \tau, k^{\bullet}, m_{1}\right) .
\end{aligned}
$$

By setting $u=x / \alpha$ in the expression (17), we obtain

$$
J\left(\alpha, \tau, k^{\bullet}, m_{1}\right)=\frac{\alpha \tau}{\Gamma\left(k^{\bullet}\right)} \int_{0}^{m_{1} / \alpha} u^{\tau k^{\bullet}} \exp \left(-u^{\tau}\right) d u .
$$

The substitution $w=u^{\tau}$ yields $J\left(\alpha, \tau, k^{\bullet}, m_{1}\right)$ in terms of the incomplete gamma function

$$
\begin{aligned}
J\left(\alpha, \tau, k^{\bullet}, m_{1}\right) & =\frac{\alpha}{\Gamma\left(k^{\bullet}\right)} \int_{0}^{\left(m_{1} / \alpha\right)^{\tau}} w^{k^{\bullet}+\tau^{-1}-1} \exp (-w) d w \\
& =\frac{\alpha}{\Gamma\left(k^{\bullet}\right)} \gamma\left[k^{\bullet}+\tau^{-1},\left(m_{1} / \alpha\right)^{\tau}\right]
\end{aligned}
$$

Hence, inserting the last result into (17) gives

$$
I\left(m_{1}\right)=\sum_{j, m, q=0}^{\infty} \frac{\alpha w_{j, m, q}(k, p, \lambda)}{\Gamma\left(k^{\bullet}\right)} \gamma\left[k^{\bullet}+\tau^{-1},\left(m_{1} / \alpha\right)^{\tau}\right] .
$$

The ExGGG mean deviations follow from (16) and the last expression. The result is analogous to $I\left(\mu_{1}^{\prime}\right)$.

\section{Reliability}

The reliability measure of the ExGGG distribution is determined considering the expression presented by Cordeiro et al. (2016), given by

$$
R=\int_{0}^{\infty} f(x) F(x) d x
$$

where $f(x)$ and $F(x)$ are calculated from (7) and (2), respectively. The reliability can be written explicitly as follows

$$
\begin{aligned}
R & =\int_{0}^{\infty} \sum_{j, m, q=0}^{\infty} w_{j, m, q}(k, p, \lambda) g_{\alpha, \tau, k^{*}}(x)\left(1-\left\{1-\frac{\gamma_{1}\left[k,\left(\frac{x}{\alpha}\right)^{\tau}\right]}{1-p\left\{1-\gamma_{1}\left[k,\left(\frac{x}{\alpha}\right)^{\tau}\right]\right\}}\right\}^{\lambda}\right) d x \\
& =\sum_{j, m, q=0}^{\infty} w_{j, m, q}(k, p, \lambda)\left(1-\int_{0}^{\infty} g_{\alpha, \tau, k^{*}}(x)\left\{1-\frac{\gamma_{1}\left[k,\left(\frac{x}{\alpha}\right)^{\tau}\right]}{1-p\left\{1-\gamma_{1}\left[k,\left(\frac{x}{\alpha}\right)^{\tau}\right]\right\}}\right\}^{\lambda} d x\right)
\end{aligned}
$$

Using the expansion (4) twice conveniently in the last expression, we have

$$
R=\sum_{j, m, q=0}^{\infty} w_{j, m, q}(k, p, \lambda)\left(1-\int_{0}^{\infty} g_{\alpha, \tau, k^{\bullet}}(x) \sum_{r, u=0}^{\infty}(-1)^{r+u}\left(\begin{array}{c}
\lambda \\
r
\end{array}\right)\left(\begin{array}{c}
-r \\
u
\end{array}\right) p^{u} \gamma_{1}\left[k,\left(\frac{x}{\alpha}\right)^{\tau}\right]^{r}\left\{1-\gamma_{1}\left[k,\left(\frac{x}{\alpha}\right)^{\tau}\right]\right\}^{u} d x\right)
$$


Using the binomial expansion in expression (18), we have

$$
R=\sum_{j, m, q=0}^{\infty} w_{j, m, q}(k, p, \lambda)\left\{1-\int_{0}^{\infty} g_{\alpha, \tau, k}(x) \sum_{r, u=0}^{\infty} \sum_{t=0}^{u}(-1)^{r+u+t}\left(\begin{array}{c}
\lambda \\
r
\end{array}\right)\left(\begin{array}{c}
-r \\
u
\end{array}\right)\left(\begin{array}{l}
u \\
t
\end{array}\right) p^{u} \gamma_{1}\left[k,\left(\frac{x}{\alpha}\right)^{\tau}\right]^{r+t} d x\right\} .
$$

We can substitute $\sum_{r, u=0}^{\infty} \sum_{t=0}^{u}$ for $\sum_{r, t=0}^{\infty} \sum_{u=t}^{\infty}$ to obtain

$$
R=\sum_{j, m, q=0}^{\infty} w_{j, m, q}(k, p, \lambda)\left\{1-\int_{0}^{\infty} g_{\alpha, \tau, k}(x) \sum_{r, t=0}^{\infty} s_{r, t}(p, \lambda) \gamma_{1}\left[k,\left(\frac{x}{\alpha}\right)^{\tau}\right]^{r+t} d x\right\},
$$

where

$$
s_{r, t}(p, \lambda)=\sum_{u=t}^{\infty}(-1)^{r+u+t}\left(\begin{array}{c}
\lambda \\
r
\end{array}\right)\left(\begin{array}{c}
-r \\
u
\end{array}\right)\left(\begin{array}{l}
u \\
t
\end{array}\right) p^{u}
$$

How $g_{\alpha, \tau, k^{\bullet}}(x)$ has distribution $\mathrm{GG}\left(\alpha, \tau, k^{\bullet}\right)$, we obtain

$$
R=\sum_{j, m, q=0}^{\infty} w_{j, m, q}(k, p, \lambda)\left\{1-\int_{0}^{\infty} \frac{\tau}{\alpha \Gamma\left(k^{\bullet}\right)}\left(\frac{x}{\alpha}\right)^{\tau k^{\bullet}-1} \exp \left[-\left(\frac{x}{\alpha}\right)^{\tau}\right] \sum_{r, t=0}^{\infty} s_{r, t}(p, \lambda) \gamma_{1}\left[k,\left(\frac{x}{\alpha}\right)^{\tau}\right]^{r+t} d x\right\} .
$$

Finally, setting $w=\left(\frac{x}{\alpha}\right)^{\tau}$ in (19), we have

$$
R=\sum_{j, m, q=0}^{\infty} w_{j, m, q}(k, p, \lambda)\left\{1-\sum_{r, t=0}^{\infty} \frac{s_{r, t}(p, \lambda)}{\Gamma\left(k^{\circ}+q\right)} I\left(k^{\circ}+q, r+t\right)\right\},
$$

where

$$
I\left(k^{\circ}+q, r+t\right)=\int_{0}^{\infty} w^{k^{\circ}+q-1} \exp (-w) \gamma_{1}(k, w)^{r+t} d w,
$$

$k^{\circ}=k(j+m+1)$ and $w_{j, m, q}(k, p, \lambda)$ is defined by expression (8). Using the Lauricella function of type A (defined in Section 4 ), the last integral can be written as

$$
I\left(k^{\circ}+q, r+t\right)=\left(k^{\circ}\right)^{-r-t} \Gamma\left[q+k^{\circ}(r+t+1)\right] \times F_{A}^{(r+t)}\left(q+k^{\circ}(r+t+1) ; k^{\circ}, \cdots, k^{\circ} ; k^{\circ}+1, \cdots, k^{\circ}+1 ;-1, \cdots,-1\right) .
$$

\section{Order Statistics}

The density function $f_{i: n}(x)$ of the $i t h$ order statistic, say $X_{i: n}$, for $i=1, \ldots, n$, from random variables $X_{1}, \ldots, X_{n}$ having density (3), is given by

$$
f_{i: n}(x)=\frac{1}{B(i, n-i+1)} f(x) F(x)^{i-1}\{1-F(x)\}^{n-i},
$$

where $f(x)$ and $F(x)$ are the pdf and cdf of the ExGGG distribution, respectively and $B(\cdot, \cdot)$ denotes the beta function. We readily obtain using the binomial expansion

$$
f_{i: n}(x)=\sum_{j_{1}=0}^{n-i} \frac{(-1)^{j_{1}}\left(\begin{array}{c}
n-i \\
j_{1}
\end{array}\right)}{B(i, n-i+1)} f(x) F(x)^{i+j_{1}-1} .
$$

However, if $F(x)$ is the cdf of ExGGG distribution defined in (2) and $u$ positive integer, we have

$$
\begin{aligned}
f(x) F(x)^{u} & =\frac{\lambda \tau(1-p)}{\alpha \Gamma(k)}\left(\frac{x}{\alpha}\right)^{\tau k-1} \exp \left[-\left(\frac{x}{\alpha}\right)^{\tau}\right]\left(1-p\left\{1-\gamma_{1}\left[k,\left(\frac{x}{\alpha}\right)^{\tau}\right]\right\}\right)^{-2}\left\{1-\frac{\gamma_{1}\left[k,\left(\frac{x}{\alpha}\right)^{\tau}\right]}{\left(1-p\left\{1-\gamma_{1}\left[k,\left(\frac{x}{\alpha}\right)^{\tau}\right]\right\}\right)}\right\}^{\lambda-1} \\
& \times\left(1-\left\{1-\frac{\gamma_{1}\left[k,\left(\frac{x}{\alpha}\right)^{\tau}\right]}{1-p\left\{1-\gamma_{1}\left[k,\left(\frac{x}{\alpha}\right)^{\tau}\right]\right\}}\right\}^{\lambda}\right)^{u} .
\end{aligned}
$$


Using the binomial expansion and (4) twice conveniently, we have

$$
\begin{aligned}
f(x) F(x)^{u} & =\frac{\lambda \tau(1-p)}{\alpha \Gamma(k)}\left(\frac{x}{\alpha}\right)^{\tau k-1} \exp \left[-\left(\frac{x}{\alpha}\right)^{\tau}\right] \sum_{l_{1}=0}^{u} \sum_{j, a=0}^{\infty}(-1)^{l_{1}+j+a}\left(\begin{array}{l}
u \\
l_{1}
\end{array}\right)\left(\begin{array}{c}
\lambda\left(l_{1}+1\right)-1 \\
j
\end{array}\right)\left(\begin{array}{c}
-(j+2) \\
a
\end{array}\right) p^{a} \\
& \times \gamma_{1}\left[k,\left(\frac{x}{\alpha}\right)^{\tau}\right]^{j}\left\{1-\gamma_{1}\left[k,\left(\frac{x}{\alpha}\right)^{\tau}\right]\right\}^{a}
\end{aligned}
$$

Now, using the binomial expansion in the expression $\left\{1-\gamma_{1}\left[k,\left(\frac{t}{\alpha}\right)^{\tau}\right]\right\}^{a}$, we obtain

$$
\begin{aligned}
f(x) F(x)^{u} & =\frac{\lambda \tau(1-p)}{\alpha \Gamma(k)}\left(\frac{x}{\alpha}\right)^{\tau k-1} \exp \left[-\left(\frac{x}{\alpha}\right)^{\tau}\right] \sum_{l_{1}=0}^{u} \sum_{j, a=0}^{\infty} \sum_{m=0}^{a}(-1)^{l_{1}+j+a+m}\left(\begin{array}{l}
u \\
l_{1}
\end{array}\right)\left(\begin{array}{c}
\lambda\left(l_{1}+1\right)-1 \\
j
\end{array}\right)\left(\begin{array}{c}
-(j+2) \\
a
\end{array}\right)\left(\begin{array}{l}
a \\
m
\end{array}\right) p^{a} \\
& \times \gamma_{1}\left[k,\left(\frac{x}{\alpha}\right)^{\tau}\right]^{j+m} .
\end{aligned}
$$

We can substitute $\sum_{a=0}^{\infty} \sum_{m=0}^{a}$ for $\sum_{m=0}^{\infty} \sum_{a=m}^{\infty}$ to obtain

$$
f(x) F(x)^{u}=\frac{\lambda \tau(1-p)}{\alpha \Gamma(k)}\left(\frac{x}{\alpha}\right)^{\tau k-1} \exp \left[-\left(\frac{x}{\alpha}\right)^{\tau}\right] \sum_{j, m=0}^{\infty} \rho_{j, m, u}(p, \lambda) \gamma_{1}\left[k,\left(\frac{x}{\alpha}\right)^{\tau}\right]^{j+m},
$$

where

$$
\rho_{j, m, u}(p, \lambda)=\sum_{l_{1}=0}^{u} \sum_{a=m}^{\infty}(-1)^{l_{1}+j+a+m}\left(\begin{array}{l}
u \\
l_{1}
\end{array}\right)\left(\begin{array}{c}
\lambda\left(l_{1}+1\right)-1 \\
j
\end{array}\right)\left(\begin{array}{c}
-(j+2) \\
a
\end{array}\right)\left(\begin{array}{l}
a \\
m
\end{array}\right) p^{a}
$$

Inserting $f(x) F(x)^{u}$ given by (21) in expression (20), applying expansion (28) and rearranging terms, the density function of the $i$ th ExGGG order statistics is expressed by

$$
f_{i: n}(x)=\sum_{j, m, q=0}^{\infty} v_{i: n, j, m, q}(k, p, \lambda) g_{\alpha, \tau, k^{*}}(x)
$$

where

$$
v_{i: n, j, m, q}=\frac{\lambda(1-p) c_{j+m, q} \Gamma\left(k^{\bullet}\right)}{B(i, n-i+1) \Gamma(k)^{j+m+1}} \sum_{j_{1}=0}^{n-i}(-1)^{j_{1}}\left(\begin{array}{c}
n-i \\
j_{1}
\end{array}\right) \rho_{j, m, i+j_{1}-1}(p, \lambda),
$$

$k^{\bullet}=k(j+m+1)+q, \rho_{j, m, i+j_{1}-1}(p, \lambda)$ is defined above and $c_{j+m, q}$ is calculated recursively by (27).

Density function (22) gives the density function of the $i$ th order statistics as a linear combination of GG densities. Hence, some of the mathematical quantities of the ExGGG order statistics can be derived by knowing those of the GG distribution. For example, the $r$ th ordinary moment and the $\operatorname{mgf} M_{i: n}(s)$ of $X_{i: n}$ are

$$
E\left(X_{i: n}^{r}\right)=\alpha^{r} \sum_{j, m, q=0}^{\infty} v_{i: n, j, m, q}(k, p, \lambda) \frac{\Gamma\left(k^{\bullet}+r / \tau\right)}{\Gamma\left(k^{\bullet}\right)}
$$

and

$$
M_{i: n}(s)=\sum_{j, m, q=0}^{\infty} v_{i: n, j, m, q}(k, p, \lambda) M_{\alpha, \tau, k} \cdot(s),
$$

where $M_{\alpha, \tau, k^{*}}(s)$ can be calculated from expression (13) or (15).

\section{Inference and Estimation}

In this Section, we discuss the maximum likelihood method and Bayesian approach for the inference and estimation of the ExGGG parameter model. We also assess the performance of the maximum likelihood method for estimating the ExGGG parameters using Monte Carlo simulation. 


\subsection{Maximum Likelihood Estimation}

Here, we consider the estimation of the parameters of the ExGGG distribution by maximum likelihood method.

Let $X_{i}$ be a random variable following (3) with the vector of parameters $\boldsymbol{\theta}=(\alpha, \tau, k, p, \lambda)^{T}$. Suppose that the data consist of $n$ independent observations $x_{i}$ of $X_{i}$ for $i=1, \ldots, n$. Parametric inference for such data are usually based on likelihood methods and their asymptotic theory. The log-likelihood $\ell(\boldsymbol{\theta})$ for the model parameters can be expressed as

$$
\begin{aligned}
\ell(\boldsymbol{\theta}) & =n \log \left[\frac{\lambda \tau(1-p)}{\alpha \Gamma(k)}\right]+(\tau k-1) \sum_{i=1}^{n} \log \left(\frac{x_{i}}{\alpha}\right)-\sum_{i=1}^{n}\left(\frac{x_{i}}{\alpha}\right)^{\tau}-2 \sum_{i=1}^{n} \log \left(1-p\left\{1-\gamma_{1}\left[k,\left(\frac{x_{i}}{\alpha}\right)^{\tau}\right]\right\}\right) \\
& +(\lambda-1) \sum_{i=1}^{n} \log \left\{1-\frac{\gamma_{1}\left[k,\left(\frac{x_{i}}{\alpha}\right)^{\tau}\right]}{1-p\left\{1-\gamma_{1}\left[k,\left(\frac{x_{i}}{\alpha}\right)^{\tau}\right]\right\}}\right\} .
\end{aligned}
$$

The score components corresponding to the parameters in $\boldsymbol{\theta}$ are

$$
\begin{aligned}
& U_{\alpha}(\boldsymbol{\theta})=-\frac{n \tau k}{\alpha}+\frac{\tau}{\alpha} \sum_{i=1}^{n} u_{i}+\frac{\tau}{\alpha \Gamma(k)} \sum_{i=1}^{n}\left(2 p q_{i}+\lambda-1\right) \frac{g_{i}}{q_{i}} u_{i}^{k} \exp \left(-u_{i}\right) \\
& U_{\tau}(\boldsymbol{\theta})=\frac{n}{\tau}+\frac{1}{\tau} \sum_{i=1}^{n}\left(k-u_{i}\right) \log u_{i}-\frac{2 p}{\tau \Gamma(k)} \sum_{i=1}^{n} g_{i} u_{i}^{k} \exp \left(-u_{i}\right) \log u_{i}-\frac{(\lambda-1)}{\tau \Gamma(k)} \sum_{i=1}^{n} \frac{g_{i}}{q_{i}} u_{i}^{k} \exp \left(-u_{i}\right) \log u_{i}, \\
& U_{k}(\boldsymbol{\theta})=-n \psi(k)+\sum_{i=1}^{n} \log u_{i}-2 p \sum_{i=1}^{n} g_{i}\left[w_{i}-\psi(k) \gamma_{1}\left(k, u_{i}\right)\right]-(\lambda-1) \sum_{i=1}^{n} \frac{g_{i}}{q_{i}}\left[w_{i}-\psi(k) \gamma_{1}\left(k, u_{i}\right)\right] \\
& U_{p}(\boldsymbol{\theta})=-\frac{n}{1-p}+2 \sum_{i=1}^{n} g_{i} q_{i}-\frac{(\lambda-1)}{1-p} \sum_{i=1}^{n} g_{i} \gamma_{1}\left(k, u_{i}\right)
\end{aligned}
$$

and

$U_{\lambda}(\boldsymbol{\theta})=\frac{n}{\lambda}+\sum_{i=1}^{n} \log \left[1-g_{i} \gamma_{1}\left(k, u_{i}\right)\right]$

where,

$$
u_{i}=\left(\frac{x_{i}}{\alpha}\right)^{\tau}, \quad q_{i}=1-\gamma_{1}\left(k, u_{i}\right), \quad g_{i}=\frac{1}{1-p q_{i}}, \quad w_{i}=\frac{\dot{\gamma}\left(k, u_{i}\right)_{k}}{\Gamma(k)}, \quad \dot{\gamma}\left(k, u_{i}\right)_{k}=\sum_{n=0}^{\infty} \frac{(-1)^{n}}{n !} J\left(u_{i}, k+n-1,1\right),
$$

$\psi($.$) is the digamma function and J\left(u_{i}, k+n-1,1\right)$ is defined in Appendix B.

The maximum likelihood estimates (MLEs) $\widehat{\boldsymbol{\theta}}$ of $\boldsymbol{\theta}$ is obtained numerically from the nonlinear equations

$$
U_{\alpha}(\boldsymbol{\theta})=U_{\tau}(\boldsymbol{\theta})=U_{k}(\boldsymbol{\theta})=U_{p}(\boldsymbol{\theta})=U_{\lambda}(\boldsymbol{\theta})=0 .
$$

For interval estimation and hypothesis tests on the model parameters, we require the $5 \times 5$ unit observed information matrix, say

$$
J=J(\boldsymbol{\theta})=\left[\begin{array}{lllll}
J_{\alpha \alpha} & J_{\alpha \tau} & J_{\alpha k} & J_{\alpha p} & J_{\alpha \lambda} \\
J_{\tau \alpha} & J_{\tau \tau} & J_{\tau k} & J_{\tau p} & J_{\beta \lambda} \\
J_{k \alpha} & J_{k \tau} & J_{k k} & J_{k p} & J_{k \lambda} \\
J_{p \alpha} & J_{p \tau} & J_{p k} & J_{p p} & J_{p \lambda} \\
J_{\lambda \alpha} & J_{\lambda \tau} & J_{\lambda k} & J_{\lambda p} & J_{\lambda \lambda}
\end{array}\right],
$$

whose elements are given in Appendix B. Under conditions that are fulfilled for parameters in the interior of the parameter space but not on the boundary, the asymptotic distribution of $\sqrt{n}(\widehat{\theta}-\boldsymbol{\theta})$ is $N_{5}\left(0, I(\boldsymbol{\theta})^{-1}\right)$, where $I(\boldsymbol{\theta})$ is the expected information matrix. This matrix can be replaced by $J(\widehat{\boldsymbol{\theta}})$, i.e., the observed information matrix evaluated $\widehat{\boldsymbol{\theta}}$. The multivariate normal $N_{5}\left(0, J(\widehat{\boldsymbol{\theta}})^{-1}\right)$ distribution can be used to construct approximate confidence intervals for the individual parameters. 
We can compute the maximum values of the unrestricted and restricted log-likelihoods to construct likelihood ratio (LR) statistics for testing some submodels (see Section 2) of the ExGGG distribution. For example, we may use LR statistics to check if the fit using the ExGGG distribution is statistically "superior" to the fit using the GGG distribution for a given data set. That is, to test $H_{0}: \lambda=1$ versus $H_{1}: \lambda \neq 1$ the LR statistics is

$$
L R=2\{\ell(\hat{\alpha}, \hat{\tau}, \hat{k}, \hat{p}, \hat{\lambda})-\ell(\tilde{\alpha}, \tilde{\tau}, \tilde{k}, \tilde{p}, 1)\},
$$

where $\hat{\alpha}, \hat{\tau}, \hat{k}, \hat{p}$ and $\hat{\lambda}$ are the MLEs under $H_{1}$ and $\tilde{\alpha}, \tilde{\tau}, \tilde{k}$ and $\tilde{p}$ are the estimates under $H_{0}$.

\subsection{Bayesian Approach}

Bayesian inference combines the likelihood of the data with the a priori distributions of the parameters, resulting in the a posteriori distribution of unknown quantities. The integration of this joint distribution is generally not analytical, requiring specialized interactive algorithms called MCMC (Markov Chain Monte Carlo) algorithms. For a Bayesian analysis, the following joint density is given a priori by

$$
\pi(\alpha, \tau, k, p, \lambda) \propto \pi(\alpha) \times \pi(\tau) \times \pi(k) \times \pi(p) \times \pi(\lambda) .
$$

Here, $\alpha \sim \Gamma\left(c_{1}, b_{1}\right), \tau \sim \Gamma\left(c_{2}, b_{2}\right), k \sim \Gamma\left(c_{3}, b_{3}\right), \lambda \sim \Gamma\left(c_{4}, b_{4}\right)$ and $p \sim \operatorname{Be}(a, b)$, where $\operatorname{Be}(a, b)$ denotes a beta distribution with mean $\frac{a}{a+b}$, variance $\frac{a b}{(a+b)^{2}(a+b+1)}$ and density function given by

$$
f(v ; a, b)=\frac{1}{\mathrm{~B}(a, b)} v^{a-1}(1-v)^{b-1},
$$

where $v \in(0,1), a>0$ and $b>0, \Gamma\left(c_{i}, b_{i}\right)$ denotes a gamma distribution with mean $c_{i} / b_{i}$, variance $c_{i} / b_{i}^{2}$ and density function given by

$$
f\left(v ; c_{i}, b_{i}\right)=\frac{b_{i}^{c_{i}} v^{c_{i}-1} \mathrm{e}^{-v \mathrm{~b}_{\mathrm{i}}}}{\Gamma\left(c_{i}\right)},
$$

where $v>0, c_{i}>0$ and $b_{i}>0$. All hyper-parameters are specified. Combining the likelihood function (23) and the prior distribution (24), the joint posterior distribution for $\alpha, \tau, k, p$ and $\lambda$ reduces to

$$
\begin{aligned}
\pi(\alpha, \tau, k, p, \lambda \mid x) & \propto n \log \left[\frac{\lambda \tau(1-p)}{\alpha \Gamma(k)}\right]+(\tau k-1) \sum_{i=1}^{n} \log \left(\frac{x_{i}}{\alpha}\right)-\sum_{i=1}^{n}\left(\frac{x_{i}}{\alpha}\right)^{\tau}-2 \sum_{i=1}^{n} \log \left(1-p\left\{1-\gamma_{1}\left[k,\left(\frac{x_{i}}{\alpha}\right)^{\tau}\right]\right\}\right) \\
& +(\lambda-1) \sum_{i=1}^{n} \log \left\{1-\frac{\gamma_{1}\left[k,\left(\frac{x_{i}}{\alpha}\right)^{\tau}\right]}{1-p\left\{1-\gamma_{1}\left[k,\left(\frac{x_{i}}{\alpha}\right)^{\tau}\right]\right\}}\right\}+\log [\pi(\alpha, \tau, k, p, \lambda)] .
\end{aligned}
$$

The posterior marginal densities of the $\alpha, \tau, k, p$ and $\lambda$ parameters are not easily obtained, because the integration of the posteriori joint density (25) is very complex. An alternative for such situations is the use of the Metropolis-Hasting algorithm, which allows simulating observations of distributions from posteriori conditional densities. For the use of the algorithm we consider the following posteriori completed conditionals for the parameters $\alpha, \tau, k, p$ and $\lambda$

$$
\begin{aligned}
& \pi(\alpha \mid x, \tau, k, p, \lambda) \propto-n \log (\alpha)+(\tau k-1) \sum_{i=1}^{n} \log \left(\frac{x_{i}}{\alpha}\right)-\sum_{i=1}^{n}\left(\frac{x_{i}}{\alpha}\right)^{\tau}-2 \sum_{i=1}^{n} \log \left(1-p\left\{1-\gamma_{1}\left[k,\left(\frac{x_{i}}{\alpha}\right)^{\tau}\right]\right\}\right) \\
&+(\lambda-1) \sum_{i=1}^{n} \log \left\{1-\frac{\gamma_{1}\left[k,\left(\frac{x_{i}}{\alpha}\right)^{\tau}\right]}{1-p\left\{1-\gamma_{1}\left[k,\left(\frac{x_{i}}{\alpha}\right)^{\tau}\right]\right\}}\right\}+\log [\pi(\alpha)], \\
& \pi(\tau \mid x, \alpha, k, p, \lambda) \propto n \log (\tau)+(\tau k) \sum_{i=1}^{n} \log \left(\frac{x_{i}}{\alpha}\right)-\sum_{i=1}^{n}\left(\frac{x_{i}}{\alpha}\right)^{\tau}-2 \sum_{i=1}^{n} \log \left(1-p\left\{1-\gamma_{1}\left[k,\left(\frac{x_{i}}{\alpha}\right)^{\tau}\right]\right\}\right) \\
&+(\lambda-1) \sum_{i=1}^{n} \log \left\{1-\frac{\gamma_{1}\left[k,\left(\frac{x_{i}}{\alpha}\right)^{\tau}\right]}{1-p\left\{1-\gamma_{1}\left[k,\left(\frac{x_{i}}{\alpha}\right)^{\tau}\right]\right\}}\right\}+\log [\pi(\tau)],
\end{aligned}
$$




$$
\begin{aligned}
\pi(k \mid x, \alpha, \tau, p, \lambda) & \propto-n \log [\Gamma(k)]+(\tau k) \sum_{i=1}^{n} \log \left(\frac{x_{i}}{\alpha}\right)-2 \sum_{i=1}^{n} \log \left(1-p\left\{1-\gamma_{1}\left[k,\left(\frac{x_{i}}{\alpha}\right)^{\tau}\right]\right\}\right) \\
& +(\lambda-1) \sum_{i=1}^{n} \log \left\{1-\frac{\gamma_{1}\left[k,\left(\frac{x_{i}}{\alpha}\right)^{\tau}\right]}{1-p\left\{1-\gamma_{1}\left[k,\left(\frac{x_{i}}{\alpha}\right)^{\tau}\right]\right\}}\right\}+\log [\pi(k)], \\
\pi(p \mid x, \alpha, \tau, k, \lambda) & \propto n \log (1-p)-2 \sum_{i=1}^{n} \log \left(1-p\left\{1-\gamma_{1}\left[k,\left(\frac{x_{i}}{\alpha}\right)^{\tau}\right]\right\}\right)+(\lambda-1) \sum_{i=1}^{n} \log \left\{1-\frac{\gamma_{1}\left[k,\left(\frac{x_{i}}{\alpha}\right)^{\tau}\right]}{1-p\left\{1-\gamma_{1}\left[k,\left(\frac{x_{i}}{\alpha}\right)^{\tau}\right]\right\}}\right\} \\
& +\log [\pi(p)]
\end{aligned}
$$

and

$\pi(\lambda \mid x, \alpha, \tau, k, p) \propto n \log (\lambda)+\lambda \sum_{i=1}^{n} \log \left\{1-\frac{\gamma_{1}\left[k,\left(\frac{x_{i}}{\alpha}\right)^{\tau}\right]}{1-p\left\{1-\gamma_{1}\left[k,\left(\frac{x_{i}}{\alpha}\right)^{\tau}\right]\right\}}\right\}+\log [\pi(\lambda)]$.

\subsection{Simulation Study}

Here, we assess the performance of the MLEs by means Monte Carlo simulation experiment with 1,000 replications. We considered no censoring case for simplicity. All results were carried out using the statistical software package R. For maximizing the log-likelihood function, we used the subroutine optim.

The evaluation of point estimation was performed based on the following quantities for each sample size: the empirical mean and the mean squared error (MSE). We set the sample size at $n=30,100,200,400$ and 800 and considered some different values for the parameters $\alpha, \tau, k, p$ and $\lambda$. The empirical results are given in Table 2 .

All results in Table 2 indicate that the estimates are close to the true values of the parameters for these sample sizes. Additionally, as the sample size increases, the MSEs decrease as expected, which means that the maximum likelihood method can be used effectively for estimating the parameters of the ExGGG distribution.

\section{Application: Permanence Time Data in Japan}

In this section, we provide one application to real data sets to illustrate the importance and flexibility of the proposed distribution using both MLEs and Bayesian approaches. The data come from a study on the permanence time in Japan of the Brazilian immigrants.

The data (Table 3) were obtained from an electronic survey (e-survey) according to the method developed by Babbie (1999), which serves to obtain data on the characteristics, actions or opinions of groups using the Internet as a research tool. The survey was carried out in the first half of 2010, by means of a reserved site with limited access, by which 246 respondents filled out questionnaires. But only 147 were used in the analysis because some immigrants were of other nationalities. We considered the main variable of interest as permanence time in Japan in years, counted from the first arrival date until the research date. Table 4 gives some descriptive statistics for these data, which include mean, variance, among others. Figure 4(a) display the histogram for these data that has bimodal shape.

By using MLEs method, we fit the ExGGG, ExWG, GGG (Ortega et al., 2011), WG (Barreto-Souza et al., 2011), GG (Stacy, 1962), Weibull (Weibull, 1951), gamma and exponential distributions to these data. To obtain the MLEs of the model parameters, we use the NLMixed procedure in SAS. Table 5 lists the MLEs (and the corresponding standard errors in parentheses) of the model parameters.

The Akaike Information Criterion (AIC), Bayesian Information Criterion (BIC), Corrected Akaike Information Criterion (CAIC), Anderson-Darling ( $\left.\mathrm{A}^{*}\right)$, Cramér-von Mises $\left(\mathrm{W}^{*}\right)$ and Kolmogorov-Smirnov (KS) statistics for the fitted distributions are reported in Table 6. In general, the lower values of these statistics indicate the best fit for the data. These results indicate that the ExGGG distribution has the lowest AIC, BIC, CAIC, $\mathrm{A}^{*}, \mathrm{~W}^{*}$ and $\mathrm{KS}$ values, and therefore our new model can be chosen as the best. The $\mathrm{A}^{*}$ and $\mathrm{W}^{*}$ statistics are described in details in Chen and Balakrishnan (1995).

Comparisons of the proposed distribution with some of its sub-models using LR statistics are performed in Table 7. The numbers in this table, specially the $p$-values, suggest that the ExGGG model yields a better fit to these data than the other distributions.

More information is provided by a visual comparison of the histogram and empirical cumulative distribution function of the data with the fitted ExGGG, ExWG, GGG, WG, GG, Weibull, gamma and exponential distributions. The plots of the 
Table 2. Empirical means and the MSEs in parentheses.

\begin{tabular}{|c|c|c|c|c|c|}
\hline & $\alpha=5.00$ & $\tau=6.00$ & $k=0.20$ & $p=0.50$ & $\lambda=0.30$ \\
\hline$n$ & $\hat{\alpha}$ & $\hat{\tau}$ & $\hat{k}$ & $\hat{p}$ & $\hat{\lambda}$ \\
\hline \multirow[t]{2}{*}{30} & 4.7397 & 6.0941 & 0.2651 & 0.7090 & 0.2091 \\
\hline & $(0.1303)$ & $(0.0935)$ & $(0.0187)$ & $(0.1235)$ & $(0.0161)$ \\
\hline \multirow[t]{2}{*}{100} & 4.7765 & 6.0793 & 0.2281 & 0.6413 & 0.2414 \\
\hline & $(0.1142)$ & $(0.0858)$ & $(0.0046)$ & $(0.0730)$ & $(0.0078)$ \\
\hline \multirow[t]{2}{*}{200} & 4.8194 & 6.0500 & 0.2213 & 0.6192 & 0.2570 \\
\hline & $(0.1043)$ & $(0.0912)$ & $(0.0024)$ & $(0.0526)$ & $(0.0065)$ \\
\hline \multirow[t]{2}{*}{400} & 4.8552 & 6.0425 & 0.2144 & 0.5953 & 0.2666 \\
\hline & $(0.0896)$ & $(0.0863)$ & $(0.0012)$ & $(0.0424)$ & $(0.0051)$ \\
\hline \multirow[t]{3}{*}{800} & 4.8965 & 6.0182 & 0.2086 & 0.5554 & 0.2791 \\
\hline & $(0.0797)$ & $(0.0854)$ & $(0.0007)$ & $(0.0313)$ & $(0.0042)$ \\
\hline & $\alpha=5.00$ & $\tau=0.75$ & $k=3.00$ & $p=0.90$ & $\lambda=1.50$ \\
\hline$n$ & $\hat{\alpha}$ & $\hat{\tau}$ & $\hat{k}$ & $\hat{p}$ & $\hat{\lambda}$ \\
\hline \multirow[t]{2}{*}{30} & 5.0425 & 0.8130 & 3.6342 & 0.9724 & 1.1258 \\
\hline & $(0.0894)$ & $(0.0496)$ & $(0.5990)$ & $(0.0084)$ & $(0.6416)$ \\
\hline \multirow[t]{2}{*}{100} & 5.0525 & 0.7103 & 3.4942 & 0.9395 & 1.4870 \\
\hline & (0.0939) & $(0.0253)$ & $(0.5615)$ & $(0.0071)$ & $(0.3453)$ \\
\hline \multirow[t]{2}{*}{200} & 5.0443 & 0.7188 & 3.3180 & 0.9249 & 1.5328 \\
\hline & $(0.1044)$ & $(0.0206)$ & $(0.3953)$ & $(0.0043)$ & $(0.1972)$ \\
\hline \multirow[t]{2}{*}{400} & 5.0267 & 0.7337 & 3.1780 & 0.9143 & 1.5237 \\
\hline & $(0.1026)$ & $(0.0146)$ & $(0.2455)$ & $(0.0026)$ & $(0.1243)$ \\
\hline \multirow[t]{3}{*}{800} & 5.0541 & 0.7580 & 3.0419 & 0.9025 & 1.5041 \\
\hline & $(0.1130)$ & $(0.0128)$ & $(0.1684)$ & $(0.0018)$ & $(0.0934)$ \\
\hline & $\alpha=13.00$ & $\tau=1.50$ & $k=4.50$ & $p=0.30$ & $\lambda=8.00$ \\
\hline$n$ & $\hat{\alpha}$ & $\hat{\tau}$ & $\hat{k}$ & $\hat{p}$ & $\hat{\lambda}$ \\
\hline \multirow[t]{2}{*}{30} & 14.7076 & 1.5726 & 4.1534 & 0.2586 & 9.0340 \\
\hline & (6.5288) & $(0.0269)$ & $(0.3338)$ & $(0.0036)$ & $(2.5181)$ \\
\hline \multirow[t]{2}{*}{100} & 14.4505 & 1.5857 & 4.2381 & 0.2702 & 8.8715 \\
\hline & $(6.5667)$ & $(0.0222)$ & $(0.3259)$ & $(0.0035)$ & $(2.5302)$ \\
\hline \multirow[t]{2}{*}{200} & 14.0991 & 1.5756 & 4.3265 & 0.2744 & 8.6806 \\
\hline & (6.3851) & $(0.0194)$ & $(0.3118)$ & $(0.0036)$ & $(2.4734)$ \\
\hline \multirow[t]{2}{*}{400} & 13.9935 & 1.5719 & 4.3459 & 0.2773 & 8.6047 \\
\hline & $(5.6576)$ & $(0.0160)$ & $(0.2978)$ & $(0.0035)$ & $(2.2650)$ \\
\hline \multirow[t]{3}{*}{800} & 13.7917 & 1.5632 & 4.3662 & 0.2838 & 8.4715 \\
\hline & $(4.0796)$ & $(0.0110)$ & $(0.2077)$ & $(0.0034)$ & $(1.9171)$ \\
\hline & $\alpha=1.00$ & $\tau=0.75$ & $k=15.00$ & $p=0.75$ & $\lambda=11.00$ \\
\hline$n$ & $\hat{\alpha}$ & $\overline{\hat{\tau}}$ & $\hat{k}$ & $\hat{p}$ & $\hat{\lambda}$ \\
\hline \multirow[t]{2}{*}{30} & 1.0799 & 0.7773 & 15.5810 & 0.7425 & 11.6440 \\
\hline & $(0.0289)$ & $(0.0065)$ & $(2.8400)$ & $(0.0191)$ & $(2.2827)$ \\
\hline \multirow[t]{2}{*}{100} & 1.0462 & 0.7533 & 15.1761 & 0.7572 & 11.3307 \\
\hline & $(0.0223)$ & $(0.0022)$ & $(1.1656)$ & $(0.0182)$ & (1.9069) \\
\hline \multirow[t]{2}{*}{200} & 1.0310 & 0.7490 & 15.1189 & 0.7573 & 11.2159 \\
\hline & $(0.0193)$ & $(0.0014)$ & $(0.8694)$ & $(0.0173)$ & $(1.5937)$ \\
\hline \multirow[t]{2}{*}{400} & 1.0206 & 0.7447 & 15.0150 & 0.7583 & 11.2129 \\
\hline & $(0.0170)$ & $(0.0010)$ & $(0.6154)$ & $(0.0162)$ & (1.0866) \\
\hline \multirow[t]{2}{*}{800} & 1.0146 & 0.7468 & 14.9577 & 0.7516 & 11.1645 \\
\hline & $(0.0139)$ & $(0.0008)$ & $(0.4588)$ & $(0.0146)$ & $(0.5790)$ \\
\hline
\end{tabular}

estimated density and cumulative distribution functions are displayed in Figures 4 and 5. Clearly, the ExGGG distribution provides a closer fit to the histogram and empirical cumulative distribution function.

For Bayesian estimation of the parameters of the ExGGG distribution, the following distributions were considered a priori: $\alpha \sim \Gamma(0.01,0.01), \tau \sim \Gamma(0.01,0.01), k \sim \Gamma(0.01,0.01), p \sim \operatorname{Be}(0.5 ; 0.5)$ and $\lambda \sim \Gamma(0.01,0.01)$. The estimation was done 
Table 3. Permanence time (years) in Japan of the Brazilian immigrants $(n=147)$.

\begin{tabular}{ccccccccccccccc}
\hline 1 & 1 & 1 & 2 & 2 & 2 & 3 & 3 & 3 & 3 & 3 & 3 & 3 & 4 & 4 \\
4 & 4 & 4 & 4 & 5 & 5 & 5 & 5 & 5 & 5 & 5 & 5 & 5 & 6 & 6 \\
6 & 6 & 6 & 6 & 6 & 6 & 6 & 7 & 7 & 7 & 8 & 8 & 9 & 9 & 9 \\
9 & 10 & 10 & 10 & 10 & 10 & 10 & 10 & 10 & 11 & 12 & 12 & 12 & 12 & 12 \\
13 & 13 & 13 & 13 & 13 & 13 & 13 & 13 & 13 & 13 & 14 & 14 & 14 & 14 & 14 \\
14 & 14 & 14 & 14 & 15 & 15 & 15 & 15 & 15 & 15 & 15 & 16 & 16 & 16 & 16 \\
16 & 16 & 17 & 17 & 17 & 17 & 17 & 18 & 18 & 18 & 18 & 18 & 18 & 18 & 18 \\
18 & 18 & 18 & 18 & 18 & 18 & 18 & 18 & 18 & 18 & 19 & 19 & 19 & 19 & 19 \\
19 & 19 & 19 & 19 & 19 & 19 & 19 & 20 & 20 & 20 & 20 & 20 & 20 & 20 & 20 \\
20 & 20 & 20 & 20 & 21 & 21 & 21 & 21 & 22 & 22 & 22 & 22 & & & \\
\hline
\end{tabular}

Table 4. Descriptive statistics of the permanence time in Japan.

\begin{tabular}{ccccc}
\hline Minimum & Mean & Median & Maximum & Variance \\
1.00 & 12.81 & 14.00 & 22.00 & 37.78 \\
\hline
\end{tabular}

Table 5. MLEs of the model parameters for the permanence time data in Japan and the corresponding SEs in parentheses.

\begin{tabular}{lccccc}
\hline Model & $\hat{\alpha}$ & $\hat{\tau}$ & $\hat{k}$ & $\hat{p}$ & $\hat{\lambda}$ \\
\hline ExGGG & 17.6851 & 12.0731 & 0.1610 & 0.9044 & 0.2283 \\
& $(0.0219)$ & $(0.0683)$ & $(0.0014)$ & $(0.0305)$ & $(0.0232)$ \\
ExWG & 10.2767 & 3.8861 & 1 & 0.9851 & 0.1169 \\
& $(0.0030)$ & $(0.0001)$ & & $(0.0066)$ & $(0.0111)$ \\
GGG & 345.3600 & 3.8158 & 0.6843 & 0.9998 & 1 \\
& $(0.0028)$ & $(0.7310)$ & $(0.1382)$ & $(0.0001)$ & \\
WG & 343.5700 & 2.6131 & 1 & 0.9998 & 1 \\
& $(0.0001)$ & $(0.1732)$ & & $(0.0001)$ & \\
GG & 21.9112 & 33.2664 & 0.04257 & 0 & 1 \\
Weibull & $(0.3329)$ & $(18.6065)$ & $(0.02505)$ & & \\
& 14.3931 & 2.1801 & 1 & 0 & 1 \\
Gamma & $(0.5679)$ & $(0.1553)$ & & & \\
& 4.4728 & 1 & 2.8639 & 0 & 1 \\
Exponential & $(0.5403)$ & & $(0.3165)$ & & \\
& 12.8095 & 1 & 1 & 0 & 1 \\
\hline
\end{tabular}

by using the Metropolis-Hastings algorithm in which, for each parameter, two independent chains with 100.000 iterations each were generated, by discarding the first 10.000 and by taking 10 by 10 . At the end 9.000 samples were obtained for each parameter, in each chain. The convergence of the chains was monitored by using the test of Gelman and Rubin (1992) $(\widehat{R})$, in which it was observed that all the parameters converged. Table 8 shows the posteriori mean, standard error, and the $95 \%$ highest posterior density intervals (HPD) a posteriori. The posteriori means present results similar to those obtained by the MLEs. The approximate posterior marginal density functions for the parameters are presented in Figure 6.

Considering the MLEs of ExGGG and ExWG distributions (Table 5) and its estimated survival function (Figure 5), we obtain some useful results. The estimate for the median permanence time of Brazilian immigrants in Japan is approximately equal to thirteen years and nine months for ExGGG distribution and twelve years and one months for ExWG distribution. The probability of a Brazilian to stay less than five years in Japan is $15.25 \%$ for ExGGG distribution and $17.55 \%$ for ExWG distribution. The probability of a immigrant to remain less than twenty years is $89.60 \%$ for ExGGG distribution and $87.08 \%$ for ExWG distribution.

\section{Conclusions}

We introduced a new five parameter distribution called the extended generalized gamma geometric (ExGGG) distribution 
Table 6. Goodness-of-fit statistics for the permanence time data in Japan and the corresponding p-values in parentheses.

\begin{tabular}{|c|c|c|c|c|c|c|}
\hline Model & AIC & BIC & CAIC & $\mathrm{A}^{*}$ & $\mathrm{~W}^{*}$ & KS \\
\hline ExGGG & 899.8 & 914.7 & 900.2 & $\begin{array}{c}\mathbf{0 . 5 9} \\
(0.66)\end{array}$ & $\begin{array}{c}\mathbf{0 . 0 9} \\
(0.62)\end{array}$ & $\begin{array}{c}\mathbf{0 . 0 8} \\
(0.32)\end{array}$ \\
\hline ExWG & 930.3 & 942.3 & 930.6 & $\begin{array}{c}3.17 \\
(0.02)\end{array}$ & $\begin{array}{c}0.56 \\
(0.03)\end{array}$ & $\begin{array}{c}0.13 \\
(0.01)\end{array}$ \\
\hline GGG & $1,011.1$ & $1,023.0$ & $1,011.3$ & $\begin{array}{c}7.72 \\
(<0.01)\end{array}$ & $\begin{array}{c}0.97 \\
(<0.01)\end{array}$ & $\begin{array}{c}0.19 \\
(<0.01)\end{array}$ \\
\hline WG & $1,009.1$ & $1,018.0$ & $1,009.2$ & $\begin{array}{c}8.80 \\
(<0.01)\end{array}$ & $\begin{array}{c}1.09 \\
(<0.01)\end{array}$ & $\begin{array}{c}0.21 \\
(<0.01)\end{array}$ \\
\hline GG & 905.3 & 914.2 & 905.4 & $\begin{array}{c}1.50 \\
(0.18)\end{array}$ & $\begin{array}{c}0.26 \\
(0.18)\end{array}$ & $\begin{array}{c}0.11 \\
(0.04)\end{array}$ \\
\hline Weibull & 957.4 & 963.4 & 957.5 & $\begin{array}{c}5.38 \\
(<0.01)\end{array}$ & $\begin{array}{c}0.86 \\
(<0.01)\end{array}$ & $\begin{array}{c}0.14 \\
(<0.01)\end{array}$ \\
\hline Gamma & 978.9 & 984.9 & 979.0 & $\begin{array}{c}6.21 \\
(<0.01)\end{array}$ & $\begin{array}{c}1.10 \\
(<0.01)\end{array}$ & $\begin{array}{c}0.18 \\
(<0.01)\end{array}$ \\
\hline Exponential & $1,045.8$ & $1,048.7$ & $1,045.8$ & $\begin{array}{c}16.14 \\
(<0.01)\end{array}$ & $\begin{array}{c}3.02 \\
(<0.01)\end{array}$ & $\begin{array}{c}0.23 \\
(<0.01) \\
\end{array}$ \\
\hline
\end{tabular}

Table 7. LR tests.

\begin{tabular}{lccc}
\hline Model & Hypotheses & LR statistics & $p$-value \\
\hline ExGGG vs ExWG & $H_{0}: k=1$ vs $H_{1}: H_{0}$ is false & 32.5 & $<0.01$ \\
ExGGG vs GGG & $H_{0}: \lambda=1$ vs $H_{1}: H_{0}$ is false & 113.3 & $<0.01$ \\
ExGGG vs WG & $H_{0}: \lambda=k=1$ vs $H_{1}: H_{0}$ is false & 113.3 & $<0.01$ \\
ExGGG vs GG & $H_{0}: p=0$ and $\lambda=1$ & 9.5 & 0.01 \\
& $\quad$ vs $H_{1}: H_{0}$ is false & & \\
ExWG vs WG & $H_{0}: \lambda=1$ vs $H_{1}: H_{0}$ is false & 80.8 & $<0.01$ \\
GGG vs WG & $H_{0}: k=1$ vs $H_{1}: H_{0}$ is false & 0.0 & 1.00 \\
\hline
\end{tabular}

(a)

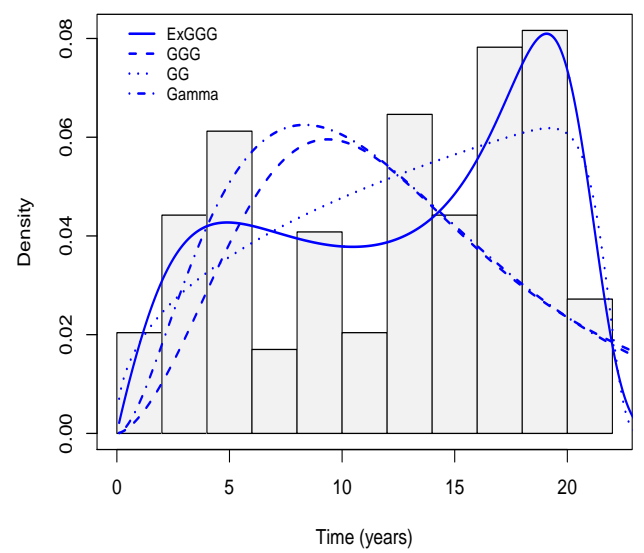

(b)

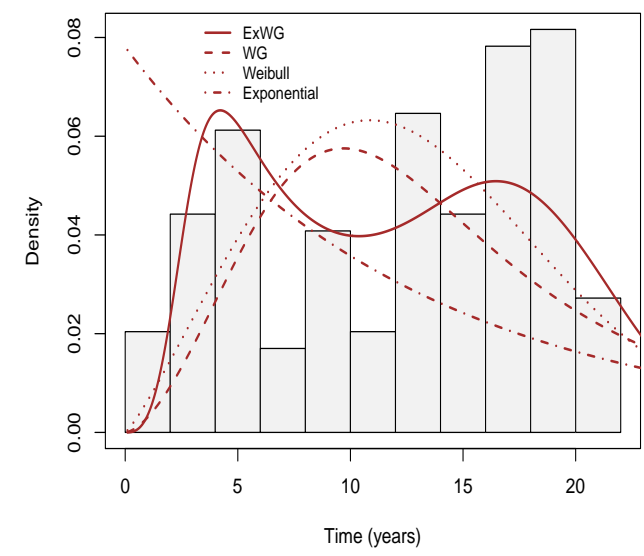

Figure 4. Histogram and fitted density functions for the permanence time data in Japan. (a) Fitted ExGGG, GGG, GG and gamma distributions. (b) Fitted ExWG, WG, Weibull and exponential distributions.

that provides a rather general and flexible framework for statistical analysis of positive data. The new distribution has positive and negative skewness (kurtosis), and further bimodal density, depending on the values of its parameters. Its hazard rate function has the forms: increasing, decreasing, in the form of bathtub and unimodal. Another important characteristic of this distribution is that it contains special sub-models such as generalized gamma geometric, Weibull geometric, expo- 
(a)

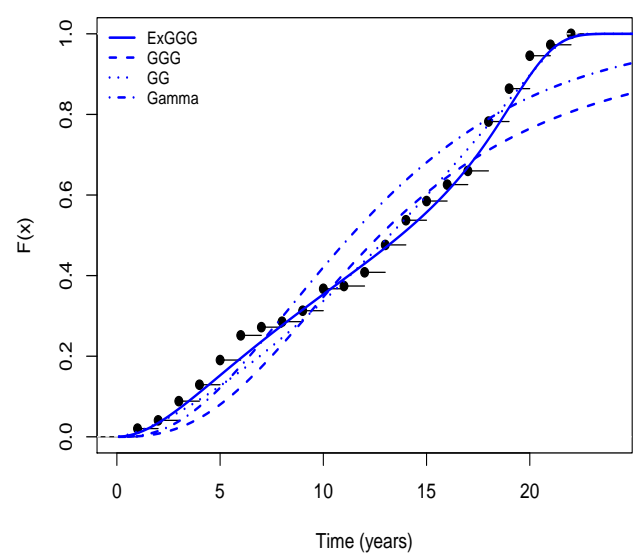

(b)

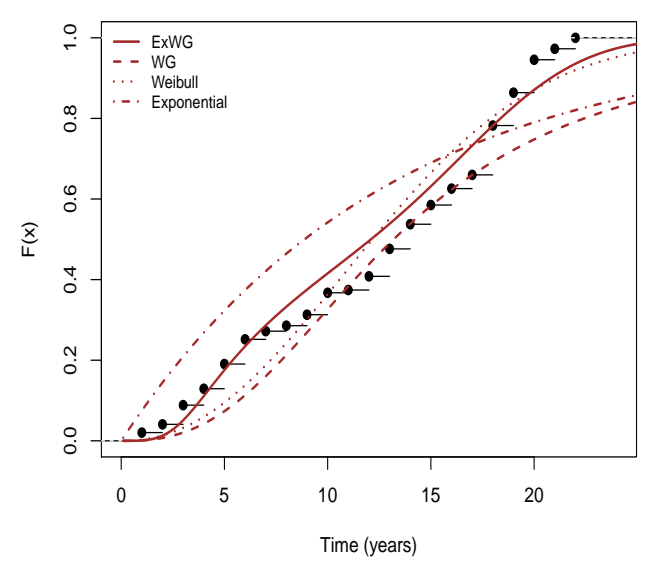

Figure 5. Estimated and empirical cumulative distribution functions for the permanence time data in Japan. (a) Fitted ExGGG, GGG, GG and gamma distributions. (b) Fitted ExWG, WG, Weibull and exponential distributions.
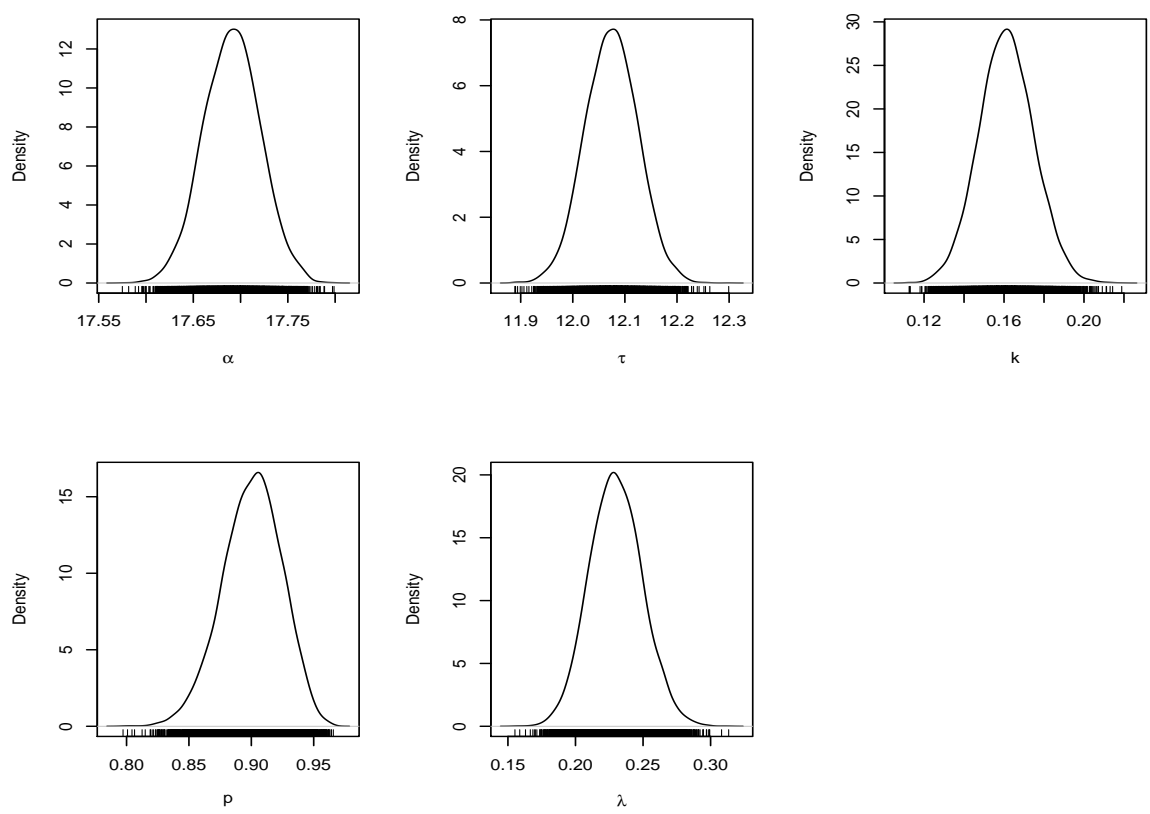

Figure 6. Approximate posterior marginal densities for the parameters from the ExGGG model for the permanence time data in Japan.

nential geometric, Rayleigh geometric, among some other distributions. Therefore the ExGGG distribution is suggested in a variety of problems for modeling lifetime data, such as bimodal and skewed. We demonstrated that the ExGGG density function can be expressed as a mixture of GG density functions. We derived explicit expressions for moments, moment generating function, mean deviations, reliability and order statistics. The estimation of parameters was approached by the method of maximum likelihood and by Bayesian method. Additionally the observed information matrix was determined. Furthermore, an application of the ExGGG distribution to real data showed that it could provide a better fit than other statistical models frequently used in lifetime data analysis. 
Table 8. Posterior summaries for the parameters from the ExGGG model for the permanence time data in Japan.

\begin{tabular}{ccccc}
\hline Parameter & Mean & SD & HPD (95\%) & $\hat{R}$ \\
\hline$\alpha$ & 17.6910 & 0.0298 & $(17.6298 ; 17.7469)$ & 1.0016 \\
$\tau$ & 12.0737 & 0.0301 & $(12.0154 ; 12.1331)$ & 1.0019 \\
$k$ & 0.1616 & 0.0129 & $(0.1363 ; 0.1864)$ & 1.0011 \\
$p$ & 0.9029 & 0.0177 & $(0.8676 ; 0.9364)$ & 1.0007 \\
$\lambda$ & 0.2292 & 0.0171 & $(0.1974 ; 0.2640)$ & 1.0001 \\
\hline
\end{tabular}

\section{References}

Aarts, R. M. Lauricella functions. (2000). From MathWorld - A Wolfram Web Resource, created by E. W. Weisstein. Available at www.mathworld.wolfram.com/LauricellaFunctions.html.

Adamidis, K., \& Loukas, S. (1998). A Lifetime distribution with decreasing failure rate. Statistics $\mathcal{E}$ Probability Letters, 39(1), 35-42. https://doi.org/10.1016/S0167-7152(98)00012-1

Babbie, E. Métodos de pesquisas de Survey. (1999). (Ed. UFMG, Belo Horizonte).

Barreto-Souza, W., de Morais, A. L., \& Cordeiro, G. M. (2011). The Weibull-geometric distribution. Journal of Statistical Computation and Simulation, 81(5), 645-657. https://doi.org/10.1080/00949650903436554

Chen, G., \& Balakrishnan, N. (1995). A general purpose approximate goodness-of-fit test. Journal of Quality Technology, 27, 154-161.

Cordeiro, G. M., Ortega, E. M. M., \& Silva, G. O. (2011). The exponentiated generalized gamma distribution with application to lifetime data. Journal of Statistical Computation and Simulation, 81(7), 827-842. https://doi.org/10.1080/00949650903517874

Cordeiro, G. M., Ortega, E. M. M., \& da Cunha, D. C. C. (2013a). The exponentiated generalized class of distributions. Journal of Data Science, 11, 1-27.

Cordeiro, G. M., Silva, G. O., \& Ortega, E. M. M. (2013b). The beta-Weibull geometric distribution. Statistics, 47(4), 817-834. https://doi.org/10.1080/02331888.2011.577897

Cordeiro, G. M., Lima, M. C. S., Cysneiros, A. H. M. A., Pascoa, M. A. R., Pescim, R. R. \& Ortega, E. M. M. (2016). An extended Birnbaum-Saunders distribution: Theory, estimation, and applications. Communications in Statistics Theory and Methods, 45(8), 2268-2297. https://doi.org/10.1080/03610926.2013.879182

Cox, C., Chu, M. F., \& Muñoz, A. (2007). Parametric survival analysis and taxonomy of hazard functions for the generalized gamma distribution. Statistics in Medicine, 26, 4352-4374. https://doi.org/10.1002/sim.2836

Exton, H. (1978). Handbook of hypergeometric integrals: theory, applications, tables, computer programs. (Halsted Press, New York).

Gelman, A., \& Rubin, D. B. (1992). Inference from iterative simulation using multiple sequences (with discussion). Statistical Science, 7, 457-472. https://doi.org/10.1214/ss/1177011136

Gradshteyn, I. S., \& Ryzhik, I. M. (2000). Table of integrals, series, and products. (Academic Press, New York).

Marshall, A. W., \& Olkin, I. (1997). A new method for adding a parameter to a family of distributions with application to the exponential and Weibull families. Biometrika, 84, 641-652. https://doi.org/10.1093/biomet/84.3.641

Nadarajah, S. (2008). Explicit expressions for moments of order statistics. Statistics and Probability Letters, 78(2), 196-205. https://doi.org/10.1016/j.spl.2007.05.022

Ortega, E. M. M., Cordeiro, G. M., \& Pascoa, M. A. R. (2011). The generalized gamma geometric distribution. Journal of Statistical Theory and Applications, 10, 433-454.

Pascoa, M. A. R., Ortega, E. M. M., \& Cordeiro, G. M. (2011). The Kumaraswamy generalized gamma distribution with application in survival analysis. Statistical Methodology, 8(5), 411-433. https://doi.org/10.1016/j.stamet.2011.04.001

Pascoa, M. A. R., de Paiva, C. M. M., Cordeiro, G. M., \& Ortega, E. M. M. (2013). The Log-Kumaraswamy generalized gamma regression model with application to chemical dependency data. Journal of Data Science, 11, 781-818.

Prudnikov, A. P., Brychkov, Y. A., \& Marichev, O. I. (1986). Integrals and Series. (Gordon and Breach Science Publishers, Amsterdam). 
Silva, R. B., Barreto-Souza, W., \& Cordeiro, G. M. (2010). A new distribution with decreasing, increasing and upsidedown bathtub failure rate. Computational Statistics and Data Analysis, 54(4), 935-944.

https://doi.org/10.1016/j.csda.2009.10.006

Stacy, E. W. (1962). A generalization of the gamma distribution. Annals of Mathematical Statistics, 33(3), 1187-1192. https://doi.org/10.1214/aoms/1177704481

Trott, M. (2006). The mathematica guidebook for symbolics. (Springer, New York).

Weibull, W. (1951). A statistical distribution function of wide applicability. Journal of Applied Mechanics, 18(3), 293297.

Wright, E. M. (1935). The asymptotic expansion of the generalized Bessel function. Proceedings of the London Mathematical Society, 38, 257-270. https://doi.org/10.1112/plms/s2-38.1.257

\section{Appendix A. Series expansion for the incomplete gamma ratio function}

Pascoa et al. (2013) developed a series expansion for the incomplete gamma ratio function given by

$$
\gamma_{1}\left[k,\left(\frac{x}{\alpha}\right)^{\tau}\right]=\frac{1}{\Gamma(k)}\left(\frac{x}{\alpha}\right)^{\tau k} \sum_{q=0}^{\infty}\left[-\left(\frac{x}{\alpha}\right)^{\tau}\right]^{q} \frac{1}{(k+q) q !} .
$$

They used an equation in Section 0.314 of Gradshteyn and Ryzhik (2000) for a power series raised to a positive integer $m$

$$
\left[\sum_{q=0}^{\infty} a_{q}\left(\frac{x}{\alpha}\right)^{\tau q}\right]^{m}=\sum_{q=0}^{\infty} c_{m, q}\left(\frac{x}{\alpha}\right)^{\tau q},
$$

whose coefficients $c_{m, q}$ (for $\left.q=1,2, \cdots\right)$ are calculated from the recurrence equation

$$
c_{m, q}=\left(q a_{0}\right)^{-1} \sum_{r=1}^{q}(m r-q+r) a_{r} c_{m, q-r}
$$

and $c_{m, 0}=a_{0}^{m}$, where $a_{q}=(-1)^{q}[(k+q) q !]^{-1}$. The coefficient $c_{m, q}$ can be obtained from $c_{m, 0}, \ldots, c_{m, q-1}$. It can also be written explicitly as functions of the quantities $a_{0}, \ldots, a_{q}$ using algebraic software such as Maple and Mathematica, although it is not necessary for programming numerically our expansions. Here, $c_{m, 0}=k^{-m}, c_{m, 1}=-m\left[(k+1) k^{m-1}\right]^{-1}$, $c_{m, 2}=m\left[2(k+2) k^{m-1}\right]^{-1}+m(m-1)\left[2(k+1)^{2} k^{m-2}\right]^{-1}$, etc. Equation (26) yields

$$
\gamma_{1}\left[k,\left(\frac{x}{\alpha}\right)^{\tau}\right]^{m}=\frac{1}{\Gamma(k)^{m}} \sum_{q=0}^{\infty} c_{m, q}\left(\frac{x}{\alpha}\right)^{\tau(k m+q)},
$$

where the coefficients $c_{m, q}$ are calculated from (27).

\section{Appendix B. Observed information matrix $J(\theta)$}

By differentiating (23), the elements of the observed information matrix $J(\boldsymbol{\theta})$ for the parameters $(\alpha, \tau, k, p, \lambda)$ are

$$
\begin{aligned}
J_{\alpha \alpha}(\boldsymbol{\theta}) & =\frac{n \tau k}{\alpha^{2}}-(\tau+1) \frac{\tau}{\alpha^{2}} \sum_{i=1}^{n} u_{i}-\frac{2 p \tau}{\alpha^{2} \Gamma(k)} \sum_{i=1}^{n} g_{i}^{2} u_{i}^{k} \exp \left(u_{i}\right) \times\left\{\left(\tau k+1-\tau u_{i}\right)\left(1-p q_{i}\right)-p\left[\frac{\tau}{\Gamma(k)} u_{i}^{k} \exp \left(u_{i}\right)\right]\right\} \\
& -\frac{(\lambda-1) \tau}{\alpha^{2} \Gamma(k)} \sum_{i=1}^{n}\left(\frac{g_{i}}{q_{i}}\right)^{2} u_{i}^{k} \exp \left(u_{i}\right)\left\{q_{i}\left(1-p q_{i}\right)\left(\tau k+1-\tau u_{i}\right)-\left[\frac{\tau}{\Gamma(k)} u_{i}^{k} \exp \left(u_{i}\right)\right]\left(-1+2 p q_{i}\right)\right\}, \\
J_{\alpha \tau}(\boldsymbol{\theta}) & =-\frac{n k}{\alpha}+\frac{1}{\alpha} \sum_{i=1}^{n} u_{i}\left(1+\log u_{i}\right)+\frac{2 p}{\alpha \Gamma(k)} \sum_{i=1}^{n} g_{i}^{2} u_{i}^{k} \exp \left(u_{i}\right)\left\{\left[1+\left(k-u_{i}\right) \log u_{i}\right]\left(1-p q_{i}\right)-\frac{p}{\Gamma(k)} u_{i}^{k} \exp \left(u_{i}\right) \log u_{i}\right\} \\
& +\frac{\lambda-1}{\alpha \Gamma(k)} \sum_{i=1}^{n}\left(\frac{g_{i}}{q_{i}}\right)^{2} u_{i}^{k} \exp \left(u_{i}\right)\left\{\left[1+\left(k-u_{i}\right) \log u_{i}\right]\left(1-p q_{i}\right) q_{i}+\frac{\left(1-2 p q_{i}\right)}{\Gamma(k)} u_{i}^{k} \exp \left(u_{i}\right) \log u_{i}\right\}, \\
J_{\alpha k}(\boldsymbol{\theta}) & =-\frac{n \tau}{\alpha}-\frac{2 p \tau}{\alpha \Gamma(k)} \sum_{i=1}^{n} g_{i}^{2} u_{i}^{k} \exp \left(u_{i}\right)\left\{\left(\psi(k)-\frac{k}{\tau} \log u_{i}\right)\left(1-p q_{i}\right)+p\left[w_{i}-\psi(k) \gamma_{1}\left(k, u_{i}\right)\right]\right\} \\
& -\frac{(\lambda-1) \tau}{\alpha \Gamma(k)} \sum_{i=1}^{n} u_{i}^{k} \exp \left(u_{i}\right)\left(\frac{g_{i}}{q_{i}}\right)^{2}\left\{\left(\psi(k)-\frac{k}{\tau} \log u_{i}\right) q_{i}\left(1-p q_{i}\right)-\left[w_{i}-\psi(k) \gamma_{1}\left(k, u_{i}\right)\right]\left(1-2 p q_{i}\right)\right\},
\end{aligned}
$$




$$
\begin{aligned}
J_{\tau \tau}(\boldsymbol{\theta}) & =-\frac{n}{\tau^{2}}-\frac{1}{\tau^{2}} \sum_{i=1}^{n} u_{i} \log ^{2} u_{i}-\frac{2 p}{\tau^{2} \Gamma(k)} \sum_{i=1}^{n} g_{i}^{2} u_{i}^{k} \exp \left(u_{i}\right) \log ^{2} u_{i} \times\left[\left(\tau-u_{i}\right)\left(1-p q_{i}\right)-\frac{p}{\Gamma(k)} u_{i}^{k} \exp \left(u_{i}\right)\right] \\
& -\frac{(\lambda-1)}{\tau^{2} \Gamma(k)} \sum_{i=1}^{n}\left(\frac{g_{i}}{q_{i}}\right)^{2} u_{i}^{k} \exp \left(u_{i}\right) \log ^{2} u_{i} \times\left[\left(\tau-u_{i}\right) q_{i}\left(1-p q_{i}\right)+\frac{\left(1-2 p q_{i}\right)}{\Gamma(k)} u_{i}^{k} \exp \left(u_{i}\right)\right],
\end{aligned}
$$$$
J_{\tau k}(\boldsymbol{\theta})=\frac{1}{\tau} \sum_{i=1}^{n} \log u_{i}-\frac{2 p}{\tau \Gamma(k)} \sum_{i=1}^{n} g_{i}^{2} u_{i}^{k} \exp \left(u_{i}\right) \log u_{i}\left\{\left[\log u_{i}-\psi(k)\right]\left(1-p q_{i}\right)-\frac{p}{\tau \Gamma(k)} u_{i}^{k} \exp \left(u_{i}\right) \log u_{i}\right\}
$$$$
-\frac{(\lambda-1)}{\tau \Gamma(k)} \sum_{i=1}^{n}\left(\frac{g_{i}}{q_{i}}\right)^{2} u_{i}^{k} \exp \left(u_{i}\right) \log u_{i}\left\{\left[\log u_{i}-\psi(k)\right] q_{i}\left(1-p q_{i}\right)+\frac{1}{\tau \Gamma(k)}\left(1-2 p q_{i}\right) u_{i}^{k} \exp \left(u_{i}\right) \log u_{i}\right\},
$$

$J_{\tau p}(\boldsymbol{\theta})=-\frac{(\lambda+1)}{\tau \Gamma(k)} \sum_{i=1}^{n} g_{i}^{2} u_{i}^{k} \exp \left(u_{i}\right) \log u_{i}$

$$
\begin{aligned}
J_{k k}(\boldsymbol{\theta}) & =-n \psi^{\prime}(k)-2 p \sum_{i=1}^{n} g_{i}^{2}\left\{\left[\frac{\ddot{\gamma}\left(k, u_{i}\right)_{k k}}{\Gamma(k)}-\gamma_{1}\left(k, u_{i}\right)\left[\psi^{\prime}(k)+\psi^{2}(k)\right]\right] \times\left(1-p q_{i}\right)-p\left[w_{i}-\psi(k) \gamma_{1}\left(k, u_{i}\right)\right]^{2}\right\} \\
& -(\lambda-1) \sum_{i=1}^{n}\left(\frac{g_{i}}{q_{i}}\right)^{2}\left(\left\{\frac{\ddot{\gamma}\left(k, u_{i}\right)_{k k}}{\Gamma(k)}-\gamma_{1}\left(k, u_{i}\right)\left[\psi^{\prime}(k)+\psi^{2}(k)\right]\right\} q_{i}\left(1-p q_{i}\right)+\left[w_{i}-\psi(k) \gamma_{1}\left(k, u_{i}\right)\right]^{2}\left(1-2 p q_{i}\right)\right),
\end{aligned}
$$

$$
J_{k p}(\boldsymbol{\theta})=-(\lambda+1) \sum_{i=1}^{n} g_{i}^{2}\left[w_{i}-\psi(k) \gamma_{1}\left(k, u_{i}\right)\right]
$$

$J_{p p}(\boldsymbol{\theta})=-\frac{n}{(1-p)^{2}}+2 \sum_{i=1}^{n} q_{i}^{2} g_{i}^{2}-\frac{(\lambda-1)}{1-p} \sum_{i=1}^{n} g_{i} \gamma_{1}\left(k, u_{i}\right)\left(1-2 p q_{i}+q_{i}\right)$,

$J_{\lambda \lambda}(\theta)=-\frac{n}{\lambda^{2}}$,

$J_{\lambda p}(\boldsymbol{\theta})=-\frac{1}{1-p} \sum_{i=1}^{n} g_{i} \gamma_{1}\left(k, u_{i}\right)$

$J_{\lambda k}(\boldsymbol{\theta})=-\sum_{i=1}^{n} \frac{g_{i}}{q_{i}}\left[w_{i}-\psi(k) \gamma_{1}\left(k, u_{i}\right)\right]$,

$J_{\lambda \tau}(\boldsymbol{\theta})=-\frac{1}{\tau \Gamma(k)} \sum_{i=1}^{n} \frac{g_{i}}{q_{i}} u_{i}^{k} \exp \left(u_{i}\right) \log u_{i}$

and

$$
J_{\lambda \alpha}(\boldsymbol{\theta})=\frac{\tau}{\alpha \Gamma(k)} \sum_{i=1}^{n} \frac{g_{i}}{q_{i}} u_{i}^{k} \exp \left(u_{i}\right),
$$

where

$$
\begin{aligned}
\dot{\gamma}\left(k, u_{i}\right)_{k} & =\sum_{n=0}^{\infty} \frac{(-1)^{n}}{n !} J\left(u_{i}, k+n-1,1\right), \\
\ddot{\gamma}\left(k, u_{i}\right)_{k k} & =\sum_{n=0}^{\infty} \frac{(-1)^{n}}{n !} J\left(u_{i}, k+n-1,2\right),
\end{aligned}
$$


$\psi^{\prime}(\cdot)$ is the derivative of the digamma function, $u_{i}, g_{i}, q_{i}$ and $w_{i}$ are defined in Section 9 . The $J(\cdot, \cdot, \cdot)$ function can be easily calculated from the integral given by Prudnikov et al. (1986) in Section 2.6.3.

$$
J(a, p, 1)=\int_{0}^{a} x^{p} \log (x) d x=\frac{a^{p+1}}{(p+1)^{2}}[(p+1) \log (a)-1]
$$

and

$$
J(a, p, 2)=\int_{0}^{a} x^{p} \log ^{2}(x) d x=\frac{a^{p+1}}{(p+1)^{3}}\{2-(p+1) \log (a)[2-(p+1) \log (a)]\} .
$$

\section{Appendix C. The SAS code for the NLMixed procedure to fit the ExGGG model.}

proc nlmixed cov data $=$ dados 1 ;

parms alpha $=11$ tau $=4.02 k=0.6 p=0.9830$ lambda $=0.10$;

bounds alpha $>0$;

bounds tau $>0$

bounds $k>0$;

bounds $p>0$;

bounds lambda $>0$;

part $1=\operatorname{probgam}((t / a l p h a) t a u, k)$;

$\log p=$ censur $*(\log (((\operatorname{lambda} * \operatorname{tau} *(1-p)) /($ alpha $* G A M M A(k))) *((t /$ alpha $) * *(\operatorname{tau} * k-1)) * \exp (-($ t/alpha $) * *(\operatorname{tau})) *$ $((1-p *(1-$ part 1$)) * *(-2)) *((1-($ part $1 *(1-p *(1-$ part 1$)) * *(-1))) * *(\operatorname{lambda}-1))))+(1-$ censur $) *(\log ((1-$ $($ part $1 *(1-p *(1-\operatorname{part} 1)) * *(-1))) * *(\operatorname{lambda})))$;

model $t \sim$ general $(\log p)$;

run;

\section{Copyrights}

Copyright for this article is retained by the author(s), with first publication rights granted to the journal.

This is an open-access article distributed under the terms and conditions of the Creative Commons Attribution license (http://creativecommons.org/licenses/by/4.0/). 